\title{
Hunting for Contact Interactions at HERA with polarized lepton and proton beams
}

\author{
J.-M. Virey ${ }^{1}$ \\ Centre de Physique Théorique*, C.N.R.S. - Luminy, Case 907 \\ F-13288 Marseille Cedex 9, France \\ and \\ Université de Provence, Marseille, France
}

\begin{abstract}
We explore the discovery and analysis potentials of the HERA collider, with and without polarized beams, in search for electron-quark contact interactions in the neutral current channel. We find that the sensitivity to contact interactions when both beams are polarized is similar to the unpolarized case, and is better than in the case where one has only lepton polarization. We emphasize that the measurement of spin asymmetries in such a polarized context could give some crucial informations on the chiral structure of these postulated new interactions. The experimental conditions are carefully taken into account.
\end{abstract}

PACS Numbers : 12.60.Cn; 13.88.+e; 13.85.Qk; 13.85.Rm

Key-Words : Contact Interaction, Polarization.

Number of figures : 3

May 1998

CPT-98/P.3541

anonymous ftp or gopher : cpt.univ-mrs.fr

*Unité Propre de Recherche 7061

${ }^{1}$ E-mail : Virey@cpt.univ-mrs.fr 


\section{Introduction}

It is commonly assumed that the Standard Model (SM) is only a low energy effective theory of a more fundamental and complete theory, which will be able to resolve naturally several problems inherent to the SM. For instance, as a non-exhaustive list of these difficulties let us mention : i) the scalar structure of the SM : nature of the Higgs boson and origin of the electroweak symmetry breaking; ii) the huge number of SM free parameters to be fixed by experiments; iii) the origin of parity violation for weak interactions; $i v$ ) the origin of the SM three generations, i.e. of the apparent replication of quarks and leptons.

Besides, the ultimate unification of all particles and of all interactions is still an essential aim of particle physicists. Then, it is natural to have several models or theories which go beyond the SM, in order to satisfy this unification goal and to resolve simultaneously some problems of the SM like those mentioned above.

The models derived from String theories or/and the Grand Unified Theories lead, in general, to the unification of the interactions. Elementary particles unification leads us to suppose that there is a deep unity between quarks and leptons and also between fermions and bosons, leading to the ideas of substructure or/and of supersymmetry.

A common prediction to all these models is the existence of new exotic particles, and, in particular, of new bosons (i.e. $Z^{\prime}$, vector and scalar leptoquarks, superpartners $\ldots)$. In the case of compositeness no particular models have emerged so far and the substructure energy scale $\Lambda$ is, in general, not directly accessible at present or planned high energy collider experiments. However, phenomenologically at some energy scale, much lower than the characteristic scale of the underlying interaction, the presence of common subconstituents follows by assuming a Contact Interaction (CI) [1, 2], independently of the details of the model. Moreover, it appears that the exchange of a new boson in a SM process can also be represented by a CI, if the boson mass $M$ is far above the center of mass energy of the elementary process (i.e. $M \gg \sqrt{\hat{s}}$ ). To the best of our knowledge,

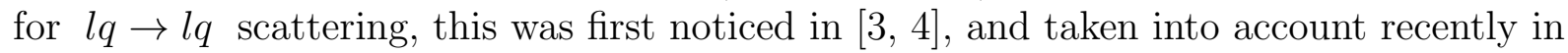
[5, 6].

So, the study of CI is a powerful way to probe new physics phenomena in general. The connexion between CI and compositeness is direct. For a new exchanged particle like a SM-like boson (i.e. $Z^{\prime}, W^{\prime}$ ) or a leptoquark-like boson (scalar or vector leptoquarks, some sfermions with $R$-parity violating couplings), the connexion is indirect in the sense that we do not consider the resonant effect.

The aim of this article is to present a purely phenomenological analysis on the experimental signatures of CI from the measurements of cross sections and spin asymmetries in the Neutral Current channel for lepton-proton collisions at HERA' This work complements previous reports [7, 8, 9, takes into account realistic experimental conditions and extends the former analysis to the two-spin case, namely when both lepton and proton beams are simultaneously polarized. The lepton polarization option was considered at

\footnotetext{
${ }^{1}$ For an unpolarized analysis in the Charged Current sector at HERA see [ [
} 
an early stage of the HERA project (see for example [10, 11]) and it is already in use at DESY with the HERMES experiment [12]. The proton polarization option at HERA [13] has been considered only recently, and it has motivated all along 1997, a workshop [15] where the main results of the present analysis have been presented [16].

Consequently, we will be interested in CI which belongs to the electron-quark sector. The conventional effective Lagrangian has then the form [7]:

$$
\begin{array}{r}
\mathcal{L}_{e q}^{N C}=\sum_{q}\left(\eta_{L L}^{q}\left(\bar{e}_{L} \gamma_{\mu} e_{L}\right)\left(\bar{q}_{L} \gamma^{\mu} q_{L}\right)+\eta_{R R}^{q}\left(\bar{e}_{R} \gamma_{\mu} e_{R}\right)\left(\bar{q}_{R} \gamma^{\mu} q_{R}\right)\right. \\
\left.+\eta_{L R}^{q}\left(\bar{e}_{L} \gamma_{\mu} e_{L}\right)\left(\bar{q}_{R} \gamma^{\mu} q_{R}\right)+\eta_{R L}^{q}\left(\bar{e}_{R} \gamma_{\mu} e_{R}\right)\left(\bar{q}_{L} \gamma^{\mu} q_{L}\right)\right)
\end{array}
$$

with $\eta_{i j}^{q}=\epsilon g^{2} /\left(\Lambda_{i j}^{q}\right)^{2}$ where $q$ indicates a quark flavour and $i, j$ correspond to different chiralities, Left (L) and Right (R). $\epsilon= \pm 1$ is a sign which characterizes the nature of the interferences of the contact terms with SM amplitudes. The coupling constant $g$ is normalized to $g^{2}=4 \pi$, since such CI have been initially invoked for compositeness studies, where the new binding forces are assumed to be strong.

If we restrict ourselves to the light quark flavours of the first generation $(i . e . q=u, d)$, we find that there are eight independent terms appearing in eq.(11). In fact, we can reduce the number of independent coefficients by the use of symmetries. For instance, the $S U(2)_{L}$ symmetry indicates the relation $\eta_{R L}^{u}=\eta_{R L}^{d}$ [8, 5, 2]. If we require the presence of some higher symmetries we will obtain similar relations among the $\eta$ coefficients, and the interested reader can find more details in references [5, 18]. Here we will not consider any particular symmetry in order to perform a "model independent" analysis. Nevertheless, for simplicity, we will assume some "universality" between the $u$ and $d$ quarks contributions, namely $\eta_{i j}^{u}=\eta_{i j}^{d}=\eta_{i j}$. However, as long as a proton beam is used at HERA, one tests essentially the structure and interactions of the $u$-quark, and more precisely its valence component.

With the assumption of " $u$ - $d$ universality", the four "chiralities" $L L, R R, L R$ and $R L$, along with the sign $\epsilon$, define eight individual models (associated to eight distinct behaviors). The CI could correspond to one of these models or to any combination of them. From now, we label each individual model by its non zero $\eta_{i j}^{\epsilon}$ coefficient, associated to the "chirality" $I J^{\epsilon}$, where we have added $\epsilon$ as an index for clarity.

The contact terms are constrained by several experiments involving electron-quark interactions, like for instance unpolarized and polarized deep inelastic scattering, Drell-Yan lepton pairs production, atomic parity violation and hadron production in $e^{+} e^{-}$collisions. A global study of the $e q$ CI for these processes, with the corresponding constraints on $\Lambda$, has been performed in [5]. In particular, the atomic parity violation experiments on Cesium atoms give some bounds of the order of $\Lambda \sim 10 \mathrm{TeV}$ for the individual models [5, 19. However, it appears that it is easy to find some combinations of the chiralities

\footnotetext{
${ }^{2}$ Note that recently it has been advocated [17, 18] that the invariance under this symmetry also imposes $\eta_{L L}^{u}=\eta_{L L}^{d}$, but this relation is no longer valid if we consider the most general $S U(2) \times U(1)$ invariant contact term Lagrangian, due to the presence of an isospin triplet exchange term [8, 5].
} 
which evade these constraints. The symmetries which are able to realize such cancellations do not necessary correspond to Parity Conserving (PC) interactions [18]. At this point, it is interesting to remark that in the framework of composite models, some naturalness arguments [20] indicate that the composite strong dynamics has to respect some unbroken chiral global symmetries, which leads to Parity Violation for the new interaction [2]. Moreover, the fact that the new interaction is Parity Violating (PV) seems to be natural in the sense that the new energy scale $\Lambda$ is above the characteristic energy scale $v \sim 246 \mathrm{GeV}$ of weak interactions, namely in an energy domain where SM PV interactions are already present [2]. In addition, if we examine the fermionic couplings of the different $Z^{\prime}$ usually involved in traditional searches (see [21] for a review), we can see that they are of PV nature, in general.

Coming back to the experimental bounds on $\Lambda$, we have noticed that the individual models are severely constrained, which is not the case when several terms of different chiralities are involved simultaneously. In this latter case, the authors of ref. [5] found that the present bounds on $\Lambda$ are of the order of $3-4 \mathrm{TeV}$. Note also that the constraints originating from atomic parity violation experiments, could also be relaxed if there are compensating contributions coming from more than one new physics source [22]. Nevertheless, for simplicity, in the following analysis we will consider the eight models individually. We will comment on the effects of some models with a more complicated chiral structure at the end of the last section.

Finally, the H1 and ZEUS collaborations at HERA have observed an excess of events, in comparison with the SM expectations, at high $Q^{2}$, in the deep inelastic positronproton cross section $\sigma_{+} \equiv d \sigma / d Q^{2}\left(e^{+} p \rightarrow e^{+} X\right)$ [23]. Many explanations have emerged since these observations (see [24] for a nice review). This excess could be interpreted as a manifestation of new physics : leptoquarks, squarks with $R$-parity violation $\left(R_{p}\right)$ or CI. In this latter case, up to some difficulties [24], it corresponds to a new interaction in the up-quark sector for a scale $\Lambda \sim 3-4 T e V$ [25, 17, 24]. Since the lepton beam is made of positrons, the cross section $\sigma\left(e^{+} p\right)$ is sensitive to the chiralities $L R^{ \pm}$and/or $R L^{ \pm}$where \pm corresponds to $\epsilon$. We can remark that the required $\Lambda$ for the HERA excess of events, is close to the present sensitivity of the other experiments studied in [0]. However, it appears that with the new data [26] "the evidence for the signal remains meager" [24]. Then we do not insist anymore on this anomaly. We will just present the different curves of our analysis for the "special" value $\Lambda=4 \mathrm{TeV}$.

We will present in section 2 our strategy of analysis and the relevant experimental parameters. In section 3, we will define the most powerful observables and discuss the discovery reaches on the CI scale $\Lambda$. At this stage, we will consider three different running experimental conditions : i) unpolarized, ii) lepton polarization only (one-spin), iii) lepton plus proton polarizations (two-spin). In section 4 we will present a strategy which could be helpful to strongly constrain the chiral structure of the possible new interaction. We give our conclusions in section 5. In appendix A, we define the double polarized cross sections, along with the expected behavior of the most relevant observables involved in this analysis. In appendix B, we present an efficient way to take into account the degrees 
of beam polarization, involved in any polarized experiments, and to define correctly the different spin asymmetries and their associated statistical errors.

\section{Observables and experiments}

\subsection{Preamble}

The effective Lagrangian described by eq.(1) is added to the SM one, and allows the calculation of the differential inclusive cross section $d \sigma_{t}^{\lambda_{e} \lambda_{p}} / d Q^{2}$ for the polarized deep inelastic scattering process :

$$
\vec{e}^{t} \vec{p} \rightarrow e^{t} X
$$

where $t$ is the electric charge of the colliding lepton and $\lambda_{e}, \lambda_{p}$ are the helicities of the lepton and the proton. We have performed the integration over the $x$ variable in order to increase the statistics, since we are concerned with the high $Q^{2}$ domain. Another reason to do that, comes from the fact that an effect due to a CI will present a continuum in the $x$-distribution $[$ The expressions for the double polarized differential cross sections are presented in appendix A.

Now, since we have defined the basic observable $d \sigma_{t}{ }^{\lambda_{e} \lambda_{p}} / d Q^{2}$, we can go into the details of our analysis. This study is divided into three parts, corresponding to three different modes of running for the HERA machine. The first one is operating presently, the other ones are in project : i) Unpolarized; ii) Lepton polarization only $\left(e^{-}\right.$and $\left.e^{+}\right)$(one-spin); iii) Lepton and proton polarizations (two-spin).

We can define several classes of observables : the cross sections (called $\sigma$ ) and the spin asymmetries. The "pure" spin asymmetries, defined for each type of lepton separately, are noted $A$. If both electron and positron beams are available, we can define a third class of observables : the charge asymmetries (called $B$ ). We put in this class the "mixed" asymmetries which are simultaneously spin and charge asymmetries.

In principle, it should be possible to get the same degree of polarization for $e^{-}$and $e^{+}$ beams. However some technical problems may arise and it could be interesting to know the separate informations coming from each lepton channel. Then we have decomposed our results into four classes : i) only the $e^{-}$beam is polarized; ii) only the $e^{+}$beam is polarized; iii) both $e^{-}$and $e^{+}$beams are polarized, but we take into account only the pure spin asymmetries $A ; i v)$ both $e^{-}$and $e^{+}$beams are polarized, but now we take into account all the possible spin and charge asymmetries ( $A$ and $B$ types). The distinction between the last two possibilities has a technical origin. It comes from the fact that we expect stronger systematic errors for the $B$-like asymmetries than for the $A$-like one's (see below).

\footnotetext{
${ }^{3}$ For a leptoquark or a $R_{p}$-squark of accessible mass at HERA, the $x$-distribution will be crucial to observe the resonance effect, since such particles are produced in the $s$-channel.
} 


\section{$2.2 \quad$ Experimental parameters}

We present in this subsection, the parameters relevant to our analysis. In order to preserve the clarity of the physical results presented in the next section, we discuss here the effects due to some reasonable variations of each parameter. Consequently, this part is rather technical and could be skipped in a first reading. Note also that the physical results only, have been given in [16].

\section{- Energy :}

We have chosen $\sqrt{s}=300 \mathrm{GeV}$ since it is the present value for the HERA machine. The optimal choice $\sqrt{s}=314 \mathrm{GeV}$ affects slightly our results, more precisely, the forthcoming bounds have to be increased by roughly $2 \%$. This is in agreement with the scaling law [9, 27]: $\Lambda_{l i m} \sim(s . L)^{\frac{1}{4}}, L$ being the integrated luminosity.

\section{- Integrated Luminosity :}

We have considered the high luminosity option [13], 15], which corresponds to a "total" integrated luminosity $L_{t o t}=1 \mathrm{fb}^{-1}$, for electrons $\left(L_{-}\right)$plus positrons $\left(L_{+}\right)$. When the beams are polarized, we have decomposed the respective luminosities according to the spin configurations in order to keep $L_{\text {tot }}$ constant, allowing us to make a realistic comparison between the "discovery potentials" of the three distincts 0,1,2-spin analysis. Namely, it gives : i) unpolarized : $L_{-}=L_{+}=1 \times 500 \mathrm{pb}^{-1}$; ii) one-spin : $L_{-}=L_{+}=2 \times 250 \mathrm{pb}^{-1}$; iii) two-spin : $L_{-}=L_{+}=4 \times 125 p b^{-1}$. In short, we take for example an integrated luminosity of $125 \mathrm{pb}^{-1}$ for the configuration $e^{-}\left(\lambda_{e}=+1\right) p\left(\lambda_{p}=+1\right)$ associated to the cross section $\sigma_{-}^{++}$, where we use the notation $\sigma_{t}^{\lambda_{e} \lambda_{p}} \equiv d \sigma_{t}^{\lambda_{e} \lambda_{p}} / d Q^{2}$.

The choice $L_{t o t}=1 \mathrm{fb}^{-1}$ could be too high. Then we have also considered the case where it is divided by a factor two. It appears that the limits on $\Lambda$ decrease by about $15 \%$, which is a little bit lower than the $\sqrt[4]{2}$ factor $(\sim 19 \%)$ indicated by the scaling law mentioned above.

\section{- Kinematic variables :}

Concerning the $y$ variable (definition in appendix A), we take the usual minimal cut [28] $y_{\min }=0.01$ (this choice has almost no influence on our results). The maximal cut is fixed to $y_{\max }=0.95$, a value which could be reached experimentally in the future at HERA [29]. If we use the present sensitivity [28] (i.e. $y_{\max }=0.8$ ), all the limits decrease by $5 \%$.

Concerning the $Q^{2}$ resolution and the corresponding minimal cut we take the ones of the ZEUS collaboration [28]: $\Delta Q^{2} / Q^{2}=34.3 \%$ and $Q_{m i n}^{2}=200 \mathrm{GeV}^{2}$. These precise choices have no visible impact on the results.

\section{- Parton distribution functions :}

For the unpolarized study we have used the three following unpolarized parton distribution functions (pdf) sets : GRV [30], MRS [31] and BS [32]. The $\Lambda$ bounds are obtained 
with the GRV set, but the other distributions give tiny differences.

For the polarized studies we have used again three polarized pdf sets : BS [32], GRSV [33] and GS96 [34]. However, the uncertainties on the polarized pdf's are presently much larger than on the unpolarized one's. These uncertainties will be strongly reduced in the near future, thanks to spin asymmetries measurements at the RHIC-BNL polarized $p p$ collider [35], for photon, jet and $W^{ \pm}$productions [36].

Consequently, we have calculated the cross sections and the asymmetries with one pdf set, then we have obtained the bounds on $\Lambda$ assuming that the uncertainties due to the pdf will be weak when the polarized HERA will be running. In other words, we have assumed that anomalous effects due to new physics will not be diluted by the uncertainties on pdf's.

The limits and the curves which will be presented, are obtained with the GRSV set since it corresponds to the most conservative attitude. Indeed, for this set the (valence) quarks are the more weakly polarized, in comparison to GS96 and BS sets, so it gives smaller spin effects and then smaller bounds. Nevertheless, we can remark that the variations on the $\Lambda_{e q}$ bounds from the different pdf sets $(\leq 3 \%)$ are weaker in comparison to the variations on the $\Lambda_{q q}$ bounds obtained from jet productions in hadron-hadron collisions $(\leq 10 \%)$ [37, 38].

\section{- Degree of beam polarization :}

From an experimental point of view, the particle beams are never fully polarized. We have to introduce a degree of polarization $P$ for each beam. The first effect is to produce a "shift" between the magnitude of the asymmetries defined theoretically $(P=1)$ and measured experimentally $(P \neq 1)$. This induces a problem of definition for the statistical error. We will comment on this difficulty in a next paragraph, and in more details in appendix B. A second effect is related to the uncertainty on the precise value of $P$, which induces a systematic error for the asymmetries.

In the following we take for the degree of beam polarization of the charged leptons $\left(P_{e}\right)$ and of the protons $\left(P_{p}\right)$, the values which are in common use [13, 15]: $P_{e^{-}}=P_{e^{+}}=$ $P_{p}=70 \%$. The choice $P_{\text {all }}=60 \%$ decreases the bounds for the one-spin and the two-spin PV asymmetries by roughly 5-6\%, and the ones for the two-spin PC asymmetries by roughly $8 \%$. It comes from the fact that the statistical errors of the PV asymmetries are proportional to $1 / P$ (see [39] for $A_{L}$ and eq.(B.19) for $A_{L L}^{P V}$ ), and those of the PC asymmetries, are proportional to $1 / P^{2}$ (see eq.(B.26)).

\section{- Systematic errors on cross sections :}

For the unpolarized cross sections, the systematic errors are rather weak and of the order of $2-5 \%$ 28]. The main uncertainties come from luminosities measurements which produce an unknown normalization factor $f[9,28]$. In the present analysis we do not take

\footnotetext{
${ }^{4}$ There are some larger values in the low $Q^{2}$ region due to energy calibration [28], but we are not concerned with this domain of $Q^{2}$.
} 
explicitly into account such a factor $f$ and its related error $\Delta f(\sim 5 \%)$, like in Martyn's analysis [9, 40], but we rather follow a procedure which is also convenient for the study of the asymmetries. Namely, we take an explicit systematic error on the involved observable, which is added in quadrature to the statistical error of the same observable. The resulting total error is used in the $\chi^{2}$ function (defined below).

For instance, we have chosen the systematic errors on the unpolarized cross sections to be $\Delta \sigma_{\text {syst }}^{\text {unpol }} / \sigma^{\text {unpol }}=3 \%$, for the entire $Q^{2}$ domain. With this choice we recover to a good accuracy the limits on $\Lambda$ presented in [28]. However, this difference in the analysis strategy in comparison to [40], gives some discrepancies on the $\Lambda$ bounds for destructive interferences $(\epsilon=-1)$, obtained from the analysis of the unpolarized cross sections. For this special case, we find some limits which are roughly $15-20 \%$ higher than the one's presented by Martyn in [40]. Nevertheless, we have to remark that this discrepancy in strategy to take into account the systematic errors, disappears in the polarized analysis since this normalisation uncertainty is irrelevant for an asymmetry, which is a ratio of cross sections.

Concerning the polarized cross sections, there are some additional systematic errors which are stronger in magnitude than for unpolarized cross sections. They arise, on the one hand, from the uncertainties in the degree of polarization, and on the other hand, from variations in counting rate due to time variations in detector efficiencies, beam intensities, and crossing angles between the different spin configurations (see 41 for more details on these systematics). These errors are added, so it is difficult to have a global estimate. This is the reason why we prefer to study spin asymmetries where these uncertainties largely cancel in the difference of cross sections, which occurs in the numerator of these asymmetries.

Without systematic errors, these polarized cross sections are the most sensitive observables to the presence of new physics. However, this "power of discovery" is quickly destroyed by systematic errors. Then, it is irrelevant to give some constraints on $\Lambda$ coming from the study of polarized cross sections if we don't have any estimate for $\Delta \sigma_{s y s t}^{p o l} / \sigma^{\text {pol }}$. In this spirit, we have estimated the values for $\Delta \sigma_{\text {syst }}^{\text {pol }} / \sigma^{\text {pol }}$ for which, the bounds on $\Lambda$ obtained from the analysis of polarized cross sections are at the same level than the ones obtained from the analysis of spin asymmetries. It appears that they have to be of the order of $10 \%$ (8\%) in the one-spin (two-spin) case.

\section{- Systematic errors on the asymmetries:}

The use of asymmetries as observables in the analysis is motivated by the minimisation of systematic errors. On the one hand, the experimental systematic errors compensate in the numerator, as we have noted just above. On the other hand, the theoretical uncertainties in cross sections, coming from higher order corrections, which are expressed in general in term of a $\mathrm{K}$ factor, partially cancel in the asymmetries [39], which are cross sections ratios.

Unfortunately, these cancellations are not exact. Moreover, our numerical simulations 
are realized at the Leading Order. Consequently, we have considered a global systematic error on the asymmetries of the order of $10 \%$ :

$$
\frac{\Delta A_{\text {syst }}}{A}=\frac{\Delta B_{\text {syst }}}{B}=10 \% \text {. }
$$

This value is the one expected at HERA in the polarized mode [41].

It appears that one can reduce this value by frequent reversals of spin orientations, a method which will be used at RHIC [35]. At HERA, it is difficult to flip the lepton spin [41] and then, this procedure is not very helpful to reduce the systematic errors. Conversely, the spin flip of the proton beam will be helpful and then we expect the smallest systematic errors for the PC asymmetries, defined below.

Concerning the charge asymmetries (B) we expect stronger systematics (i.e $>10 \%)$ since we need data from runs in the $e^{-}$mode and in the $e^{+}$mode, which cannot be done simultaneously.

Finally, we have considered the impact of the variation of this systematic error. It appears that the choice $\Delta \mathcal{O}_{\text {sys }} / \mathcal{O}=20 \%(\mathcal{O}=A$ or $B)$ decreases the bounds by roughly $10-15 \%$, the precise value depending on the involved asymmetry.

\section{- Statistical errors on the asymmetries :}

When we take into account the degrees of polarization of the beams, the definition of the statistical error of the corresponding asymmetry may be ambiguous. For the spin asymmetries which have been studied/measured until now, like the PV one-spin asymmetry $A_{L}\left(\equiv A_{L R}\right)$ or the PC two-spin asymmetries $A_{L L}^{P C}$ and $A_{\|}$, defined in deep inelastic scattering at low $Q^{2}$, the difficulty for the definition of $\Delta A_{\text {stat }}$ does not appear due to natural factorization $\left(A_{L}, A_{L L}^{P C}\right)$ or relevant assumptions $\left(A_{\|}\right)$. (These asymmetries will be defined in the next section or in appendix B). In this paper, we consider a large set of spin asymmetries where such natural factorizations do not hold generally. Moreover, since we are concerned with the high $Q^{2}$ domain, we cannot make some assumptions, like parity conservation relevant at low energy where, for instance, $A_{\|}$measurements are realized.

However, this problem is relatively technical and we prefer to insist on the physical results that will be presented in the next sections, then we give the definition of $\Delta A_{\text {stat }}$ in appendix B.

\section{- $\underline{\chi^{2} \text { analysis }}$ :}

The bounds on the scale $\Lambda$ are obtained with a $\chi^{2}$ analysis where the SM is the reference. The $\chi^{2}$ fonction is defined by :

$$
\chi^{2}=\sum_{Q^{2}}\left(\frac{\mathcal{O}^{S M+N P}\left(Q^{2}\right)-\mathcal{O}^{S M}\left(Q^{2}\right)}{\Delta \mathcal{O}^{S M}\left(Q^{2}\right)}\right)^{2},
$$

where $\mathcal{O}$ is the involved observable, the indices $S M$ and $S M+N P$ refer respectively, to the values taken by $\mathcal{O}$ in the $\mathrm{SM}$ alone and in the SM with the New Physics (CI) 
contributions. $\Delta \mathcal{O}$ is the quadratic sum of the statistical and systematic errors on $\mathcal{O}$. $\sum_{Q^{2}}$ corresponds to the sum over all the $Q^{2}$ bins defined in our analysis.

We can also add to this function a sum over all the independent observables $\mathcal{O}$. However, it appears that it is not actually relevant in our analysis since we consider for the CI, only one "individual" model at once, whose contribution is, almost, mesurable in only one double polarized cross section. This statement has to be reconsidered in the case of models involving several "chiralities" (i.e. several $\eta_{i j}^{\epsilon}$ ).

The presence of a CI will induce an increase (from 0 ) of the $\chi^{2}$ function. If we assume that no effects are detected, we obtain a limit on $\Lambda$ at the $95 \%$ Confidence Level (CL), if the $\chi^{2}$ increases, for this $\Lambda$, by $(1.96)^{2}$ 42]. In the following all the bounds which are presented correspond to a $95 \%$ CL.

\section{Definitions of the observables and $\Lambda_{e q}$ bounds}

\subsection{Unpolarized case}

The "basic" observables are the two unpolarized cross sections $\sigma_{-}$and $\sigma_{+},\left(\sigma_{t} \equiv d \sigma_{t} / d Q^{2}\right)$. With these two cross sections we can define the first (unpolarized) charge asymmetry, which was also considered in [7, 9]:

$$
B_{o}=\frac{\sigma_{-}-\sigma_{+}}{\sigma_{-}+\sigma_{+}}
$$

Using these observables, with the experimental parameters and the $\chi^{2}$ analysis described above, we obtain the bounds on $\Lambda$ presented in Table 3.1. For each row, the relevant observable is mentioned, and $\epsilon=+1(\epsilon=-1)$ corresponds to constructive (destructive) interferences.

\begin{tabular}{|c|c||c|c|c|c||c|c|c|c|}
\hline$\Lambda(\mathrm{TeV})$ & Observable(s) & $\eta_{L L}^{+}$ & $\eta_{R R}^{+}$ & $\eta_{L R}^{+}$ & $\eta_{R L}^{+}$ & $\eta_{L L}^{-}$ & $\eta_{R R}^{-}$ & $\eta_{L R}^{-}$ & $\eta_{R L}^{-}$ \\
\hline \hline$e^{-}$only & $\sigma_{-}$ & 6.2 & 6.0 & 2.6 & 2.8 & 5.4 & 5.2 & 1.8 & 1.8 \\
\hline$e^{+}$only & $\sigma_{+}$ & 3.3 & 3.4 & 6.2 & 6.0 & 2.6 & 2.7 & 5.2 & 5.0 \\
\hline$e^{-}$and $e^{+}$ & $\sigma_{-}+\sigma_{+}$ & 6.3 & 6.1 & 6.2 & 6.0 & 5.5 & 5.3 & 5.2 & 5.0 \\
\hline$e^{-}$and $e^{+}$type $B$ & $B_{o}$ & 3.1 & 2.9 & 4.7 & 4.3 & 3.1 & 2.8 & 3.8 & 3.3 \\
\hline
\end{tabular}

Table 3.1: Limits on $\Lambda$ at $95 \%$ CL for the unpolarized case.

The conclusions from this first set of results are the followings :

- Depending on the lepton type, the cross sections are sensitive to certain chiralities : $\sigma_{-}\left(\sigma_{+}\right)$tests the chiralities $L L^{ \pm}, R R^{ \pm}\left(L R^{ \pm}, R L^{ \pm}\right)$. (This confirms our expectations from our analysis of the dominant terms performed in appendix A). If a

\footnotetext{
${ }^{5}$ This remark is valid for some Gaussian statistics. At very high $Q^{2}$, where the numbers of events are small, we have used a Poisson statistics in agreement with 42].
} 
non-standard effect is observed at HERA, the comparison of the two cross sections $\sigma_{-}$and $\sigma_{+}$allows the distinction of the two classes of chiralities $\left(L L^{ \pm}, R R^{ \pm}\right)$and $\left(L R^{ \pm}, R L^{ \pm}\right)$. Note that unpolarized cross sections measurements are unable to discriminate within each classes, except if the destructive interferences pattern is actually seen, which seems to be difficult according to the experimental conditions (see next section).

- For constructive interferences we are sensitive to $\Lambda\left(\eta_{i j}^{+}\right) \sim 6 \mathrm{TeV}$, and to $\Lambda\left(\eta_{i j}^{-}\right) \sim$ $5 \mathrm{TeV}$ for destructive interferences. See 40 for comparison up to a factor 2 in integrated Luminosity (i.e. using the scaling law given in the preceding section for a relevant comparison).

- The compilation of $e^{-}$and $e^{+}$data does not increase significantly the limits on $\Lambda$ for a given chirality, but it allows the test of all the chiralities.

- The measurement of the charge asymmetry $B_{o}$, does not give any complementary informations.

The behavior of these updated results is analogous to the one's obtained some years ago in [7, 9].

\subsection{One-spin case}

The "basic" observables are the four single polarized cross sections $\sigma_{-}^{-}, \sigma_{-}^{+}$and $\sigma_{+}^{-}, \sigma_{+}^{+}$, $\left(\sigma_{t}^{\lambda_{e}} \equiv d \sigma_{t}^{\lambda_{e}} / d Q^{2}\right)$. These four cross sections allow the definition of two "pure" spin asymmetries, which are PV (i.e. non-zero for PV interactions) :

$$
A_{L}\left(e^{-}\right)=\frac{\sigma_{-}^{-}-\sigma_{-}^{+}}{\sigma_{-}^{-}+\sigma_{-}^{+}} \quad \text { and } \quad A_{L}\left(e^{+}\right)=\frac{\sigma_{+}^{-}-\sigma_{+}^{+}}{\sigma_{+}^{-}+\sigma_{+}^{+}},
$$

and four charge asymmetries, two by two independent :

$$
\begin{array}{rlrl}
B_{1}^{1} & =\frac{\sigma_{-}^{-}-\sigma_{+}^{-}}{\sigma_{-}^{-}+\sigma_{+}^{-}} & \text {and } & B_{1}^{2}=\frac{\sigma_{-}^{+}-\sigma_{+}^{+}}{\sigma_{-}^{+}+\sigma_{+}^{+}}, \\
B_{1}^{3}=\frac{\sigma_{-}^{-}-\sigma_{+}^{+}}{\sigma_{-}^{-}+\sigma_{+}^{+}} & \text {and } & B_{1}^{4}=\frac{\sigma_{-}^{+}-\sigma_{+}^{-}}{\sigma_{-}^{+}+\sigma_{+}^{-}} .
\end{array}
$$

The lower index 1 indicates that only one beam is polarized. These asymmetries have been already defined in [7, 9]. In fact, we can construct two other charge asymmetries which involve more than two independent cross sections, but only the following one appears to have an interesting discovery potential :

$$
B_{1}^{5}=\frac{\sigma_{-}^{-}-\sigma_{-}^{+}+\sigma_{+}^{-}-\sigma_{+}^{+}}{\sigma_{-}^{-}+\sigma_{-}^{+}+\sigma_{+}^{-}+\sigma_{+}^{+}} .
$$

Concerning the $\Lambda$ bounds, from the $\chi^{2}$ analysis we have obtained the results presented in Table 3.2. 


\begin{tabular}{|c|c||c|c|c|c||c|c|c|c|}
\hline$\Lambda(\mathrm{TeV})$ & Observable(s) & $\eta_{L L}^{+}$ & $\eta_{R R}^{+}$ & $\eta_{L R}^{+}$ & $\eta_{R L}^{+}$ & $\eta_{L L}^{-}$ & $\eta_{R R}^{-}$ & $\eta_{L R}^{-}$ & $\eta_{R L}^{-}$ \\
\hline \hline$e^{-}$only & $A_{L}\left(e^{-}\right)$ & 4.6 & 5.6 & 2.2 & 3.0 & 4.2 & 5.2 & 2.0 & 2.6 \\
\hline$e^{+}$only & $A_{L}\left(e^{+}\right)$ & 2.8 & 3.5 & 4.4 & 5.6 & 2.0 & 3.0 & 4.0 & 5.1 \\
\hline$e^{-}$and $e^{+}$type $A$ & $A_{L}\left(e^{-}\right)+A_{L}\left(e^{+}\right)$ & 4.7 & 5.7 & 4.4 & 5.6 & 4.3 & 5.3 & 4.0 & 5.1 \\
\hline$e^{-}$and $e^{+}$type $B$ & $B_{1}^{n}$ & $4.3^{5}$ & $5.6^{2}$ & $5.6^{2}$ & $5.4^{4}$ & $4.0^{5}$ & $5.3^{2}$ & $5.1^{2}$ & $4.7^{4}$ \\
\hline
\end{tabular}

Table 3.2: Limits on $\Lambda$ at 95\% CL for the one-spin case. For the charge asymmetries $B_{1}^{n}$ (last row), the exponent $n$ which characterizes the involved asymmetry, is also indicated for each corresponding $\Lambda$ limit value.

Some comments are in order:

- The charge asymmetries $B_{1}^{1}$ and $B_{1}^{3}$ do not appear in the last row of Table 3.2, which indicates that their sensitivity to CI are reduced in comparison to the other asymmetries.

- The $\Lambda$ bounds are much lower than in the unpolarized analysis, in particular for the $L L^{ \pm}$chirality.

- We find a smaller difference (for $\Lambda$ limit) between the two conditions of constructive and destructive interferences than in the unpolarized case.

From a "discovery potential" point of view, we conclude that the analysis of one-spin asymmetries, defined when lepton polarization is available, is less efficient than an unpolarized analysis. Such a conclusion has already been given in [9, 44]. However, if a new physics effect is detected, the analysis of one-spin asymmetries will be very useful to obtain some crucial informations on the chirality structure of the new interaction [7, 9]. It will be demonstrated in the next section for the two-spin case, since it is more powerful than the one-spin case. Indeed, for models with some "complex" chiral structure (i.e. involving several chiralities simultaneously), some cancellations may occur, which reduce considerably the "analysing potential" of the one-spin asymmetries.

The interested reader could consult [7, 9] to see the behavior of the different one-spin asymmetries.

\subsection{Two-spin case.}

The "basic" observables are the eight double polarized cross sections $\sigma_{-}^{--}, \sigma_{-}^{++}, \sigma_{-}^{-+}, \sigma_{-}^{+-}$ and $\sigma_{+}^{--}, \sigma_{+}^{++}, \sigma_{+}^{-+}, \sigma_{+}^{+-},\left(\sigma_{t}^{\lambda_{e} \lambda_{p}} \equiv d \sigma_{t}^{\lambda_{e} \lambda_{p}} / d Q^{2}\right)$.

We can construct twelve "pure" spin asymmetries involving two cross sections only. It turns out that the ones which have the greatest "analysing power" are the two PV spin asymmetries :

$$
A_{L L}^{P V}\left(e^{-}\right)=\frac{\sigma_{-}^{--}-\sigma_{-}^{++}}{\sigma_{-}^{--}+\sigma_{-}^{++}} \quad \text { and } \quad A_{L L}^{P V}\left(e^{+}\right)=\frac{\sigma_{+}^{--}-\sigma_{+}^{++}}{\sigma_{+}^{--}+\sigma_{+}^{++}} .
$$


Of course, if the new interaction is PC, these two asymmetries are irrelevant. Therefore, it is interesting to study the "discovery potential" of the PC two-spin asymmetries. In addition, they could be important to disentangle the properties of a new interaction having a more complex chiral stucture than the individual models only. These PC asymmetries, all independent, are defined by :

$$
\begin{array}{rlrl}
A_{2}^{1} & =\frac{\sigma_{-}^{--}-\sigma_{-}^{-+}}{\sigma_{-}^{--}+\sigma_{-}^{-+}} & , & A_{2}^{2}=\frac{\sigma_{-}^{++}-\sigma_{-}^{+-}}{\sigma_{-}^{++}+\sigma_{-}^{+-}}, \\
A_{2}^{3}=\frac{\sigma_{+}^{--}-\sigma_{+}^{-+}}{\sigma_{+}^{--}+\sigma_{+}^{-+}} & , & A_{2}^{4}=\frac{\sigma_{+}^{++}-\sigma_{+}^{+-}}{\sigma_{+}^{++}+\sigma_{+}^{+-}} .
\end{array}
$$

We see that they involve the cross sections with proton spin flip only. This could be particularly interesting to minimize systematic errors. Moreover, we notice that $A_{2}^{2}$ and $A_{2}^{4}$ are the spin asymmetries (usually called $A_{\|}$), which are, in general, used to extract the polarized structure function $g_{1}$. Three other spin asymmetries are of particular interest :

$$
\bar{A}_{L L}^{P V}\left(e^{-}\right)=\frac{\sigma_{-}^{-+}-\sigma_{-}^{+-}}{\sigma_{-}^{-+}+\sigma_{-}^{+-}}, \quad \bar{A}_{L L}^{P V}\left(e^{+}\right)=\frac{\sigma_{+}^{-+}-\sigma_{+}^{+-}}{\sigma_{+}^{-+}+\sigma_{+}^{+-}}, \quad A_{2}^{5}=\frac{\sigma_{-}^{++}-\sigma_{-}^{-+}}{\sigma_{-}^{++}+\sigma_{-}^{-+}} .
$$

Now, we want to introduce, among the huge number of charge asymmetries that can be defined, the ones which will have the largest discovery potential in the present analysis :

$$
\begin{gathered}
B_{2}^{1}=\frac{\sigma_{-}^{-+}-\sigma_{+}^{--}}{\sigma_{-}^{-+}+\sigma_{+}^{--}} \quad, \quad B_{2}^{2}=\frac{\sigma_{-}^{++}-\sigma_{+}^{++}}{\sigma_{-}^{++}+\sigma_{+}^{++}}, \\
B_{2}^{3}=\frac{\sigma_{-}^{--}-\sigma_{-}^{++}+\sigma_{+}^{+-}-\sigma_{+}^{-+}}{\sigma_{-}^{--}+\sigma_{-}^{++}+\sigma_{+}^{+-}+\sigma_{+}^{-+}} \quad, \quad B_{2}^{4}=\frac{\sigma_{-}^{--}-\sigma_{-}^{++}+\sigma_{+}^{--}-\sigma_{+}^{++}}{\sigma_{-}^{--}+\sigma_{-}^{++}+\sigma_{+}^{--}+\sigma_{+}^{++}} .
\end{gathered}
$$

The bounds on $\Lambda$ from these different two-spin asymmetries are presented in Table 3.3. We have to recall that these limits are strongly dependent on the systematic errors assumed for the asymmetries, and we refer to the discussion presented in section 2 concerning the expectations for $\Delta \mathcal{O}_{\text {syst }} / \mathcal{O}$ and the corresponding dependence for the different sets of asymmetries.

\begin{tabular}{|c|c||c|c|c|c||c|c|c|c|}
\hline$\Lambda(\mathrm{TeV})$ & Observable(s) & $\eta_{L L}^{+}$ & $\eta_{R R}^{+}$ & $\eta_{L R}^{+}$ & $\eta_{R L}^{+}$ & $\eta_{L L}^{-}$ & $\eta_{R R}^{-}$ & $\eta_{L R}^{-}$ & $\eta_{R L}^{-}$ \\
\hline \hline$e^{-}$only & $A_{2}^{n}\left(e^{-}\right)$ & $5.5^{P V}$ & $6.0^{P V}$ & $3.1^{5}$ & $3.4 \overline{P V}$ & $5.4^{P V}$ & $5.8^{P V}$ & $2.9^{5}$ & $3.1_{\overline{P V}}$ \\
\hline$e^{+}$only & $A_{2}^{n}\left(e^{+}\right)$ & $3.6^{4}$ & $3.9_{\overline{P V}}$ & $5.3^{P V}$ & $6.0^{P V}$ & $3.5^{4}$ & $3.6 \overline{P_{V}}$ & $5.1^{P V}$ & $5.8^{P V}$ \\
\hline$e^{-}$and $e^{+}$type $A$ & $A_{2}^{n}\left(e^{-}\right)+A_{2}^{n}\left(e^{+}\right)$ & 5.7 & 6.2 & 5.4 & 6.1 & 5.6 & 6.0 & 5.2 & 6.0 \\
\hline$e^{-}$and $e^{+}$type $B$ & $B_{2}^{n}$ & $5.5^{4}$ & $6.1^{3}$ & $6.0^{2}$ & $6.0^{1}$ & $5.5^{4}$ & $5.9^{3}$ & $5.7^{2}$ & $5.5^{1}$ \\
\hline$e^{-}$and $e^{+}$type $A$ & $A_{2}^{P C}$ & $4.6^{1}$ & $4.4^{2}$ & $4.6^{4}$ & $4.3^{3}$ & $4.8^{1}$ & $4.6^{2}$ & $4.8^{4}$ & $4.5^{3}$ \\
\hline
\end{tabular}

Table 3.3: Limits on $\Lambda$ at 95\% CL for the two-spin case. The label " $\overline{P V} "$ corresponds to the asymmetry $\bar{A}_{L L}^{P V}$.

The results have the following properties : 
- For constructive interferences, the limits are at the same level for $R R^{+}$and $R L^{+}$ models and slightly lower for $L L^{+}$and $L R^{+}$models, than the ones obtained from the unpolarized analysis. For destructive interferences the bounds are better. Roughly, we find that the limits are comparable in magnitude to the unpolarized case, and then, far better than for the one-spin case. This result is drastically different than the one obtained for quark-quark CI studies for jet production in hadron-hadron collisions. In this case, we found that spin asymmetries studies have a much greater discovery potential than unpolarized cross sections studies 38. This fact is directly correlated to the huge systematic errors associated to any unpolarized jet cross sections.

- The differences between the two conditions of constructive and destructive interferences are reduced again, but we have still $\Lambda\left(\eta_{i j}^{+}\right)>\Lambda\left(\eta_{i j}^{-}\right)$.

- Informations on the chiral structure of the new interaction could be obtained, and this, with a better sensitivity than for the one-spin case. This analysis is the aim of the following section.

\section{Chiral structure analysis}

In this section, we propose a strategy of analysis in order to obtain some precise informations on the chiral structure of an eventual new interaction, and on the nature of the interferences with the SM (i.e. sign of $\epsilon$ ).

In a first step, for simplicity, we consider only the individual models. Some remarks on the models with a more complicated structure will be given in a following subsection. The curves are given for $\Lambda=4 \mathrm{TeV}$, in connection with the possible HERA anomaly.

We have seen in the preceding section that the unpolarized cross sections are sensitive to two classes of chiralities, depending on the electric charge of the colliding lepton : $\sigma_{-}$is sensitive to $\left(L L^{ \pm}, R R^{ \pm}\right)$and $\sigma_{+}$to $\left(L R^{ \pm}, R L^{ \pm}\right)$. Then, it is necessary to run into the two channels $\left(e^{-} p\right.$ and $\left.e^{+} p\right)$ in order to cover all the possible chiralities. Moreover, experimentally in order to obtain the value of $\epsilon$, we need to identify the destructive interference pattern, which could be feasible if $\Lambda$ is small and the integrated luminosity very high. At HERA, with $L_{\text {tot }}=1 \mathrm{fb}^{-1}$, it needs $\Lambda<3.5 \mathrm{TeV}$, a case which is already excluded by Drell-Yan process analysis at the Tevatron [5].

Consequently, we cannot go further than the separation of the two classes $\left(L L^{ \pm}, R R^{ \pm}\right)$$\left(L R^{ \pm}, R L^{ \pm}\right)$from unpolarized cross sections measurements. The analysis of the different spin asymmetries allows a clear separation of the different individual models. We will illustrate this fact with the two-spin asymmetries. 


\subsection{Two-spin case}

The first spin asymmetries to consider are the PV one's $A_{L L}^{P V}\left(e^{-}\right)$and $A_{L L}^{P V}\left(e^{+}\right)$, which give the strongest constraints on $\Lambda$. They are represented on Figure 1 .
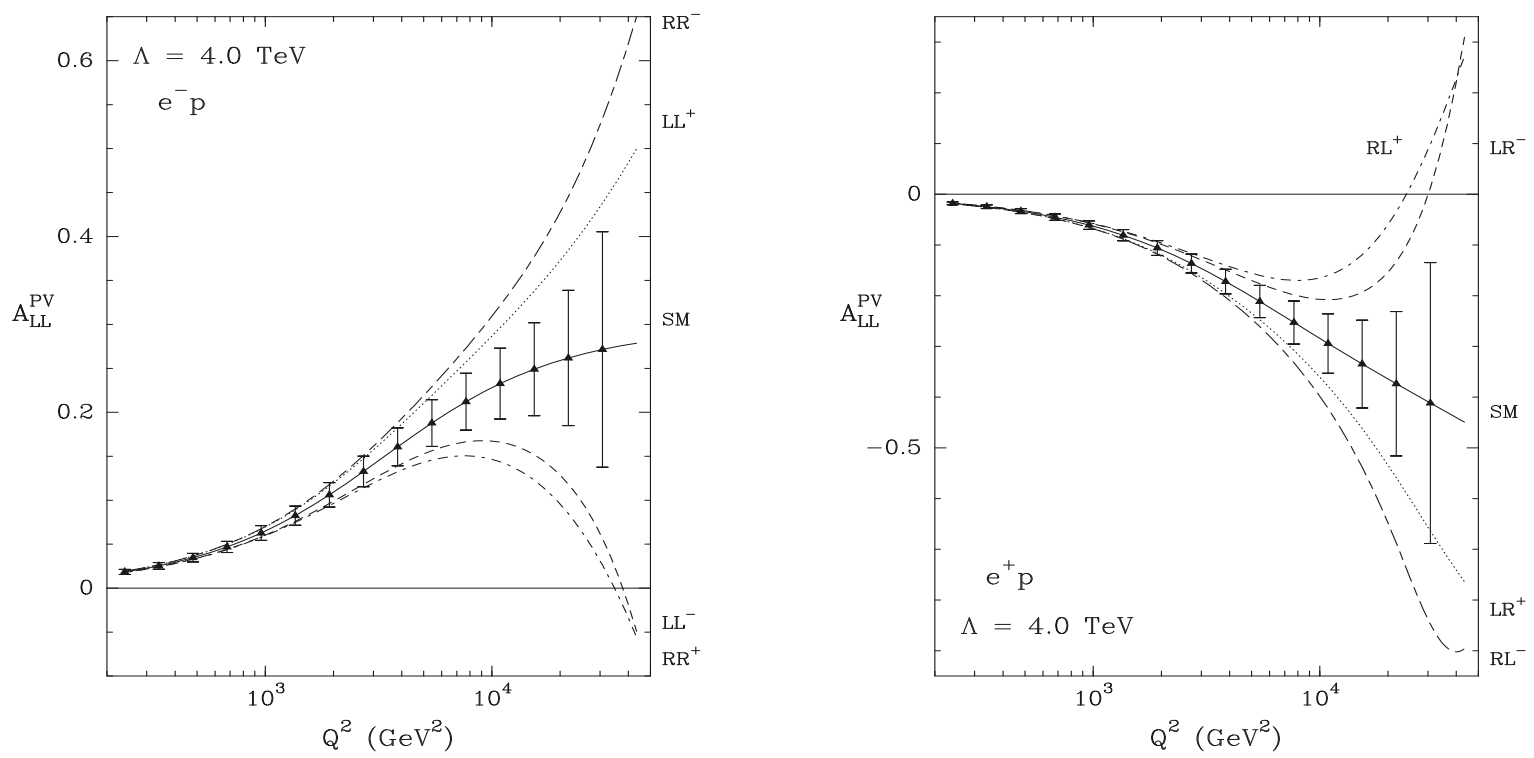

Figure 1: Spin asymmetries $A_{L L}^{P V}\left(e^{-}\right)$and $A_{L L}^{P V}\left(e^{+}\right)$. SM predictions (plain) and contact interactions for $\Lambda=4 \mathrm{TeV}$, the relevant chiralities are given on the figure. Chiralities which are too close to the SM, are omitted.

The behavior of these asymmetries is in perfect agreement with the expectations from the studies of their dominant terms, performed in appendix A, which indicate for the numerator of the asymmetries :

$$
\begin{aligned}
& \operatorname{num}\left[A_{L L}^{P V}\left(e^{-}\right)\right] \simeq \epsilon K(+\eta+\eta \prime) u^{+}, \\
& \operatorname{num}\left[A_{L L}^{P V}\left(e^{+}\right)\right] \simeq \epsilon K(-\eta+\eta \prime) u^{+},
\end{aligned}
$$

where $u^{+}$is the $u$ quark distribution for a quark helicity parallel to that of the parent proton. $K=8 \pi \alpha^{2} / 3 Q^{2} \Lambda^{2}$ and $\eta$ and $\eta^{\prime}$ characterize the chiral structure of the CI, with the convention $L L(\eta=+1, \eta \prime=+1), R R(-1,-1), L R(+1,-1), R L(-1,+1)$. Then, we deduce the following properties:

- Concerning $A_{L L}^{P V}\left(e^{-}\right)$, a deviation from the SM expectation allows to separate the class $\left(L L^{+}, R R^{-}\right)$(positive deviation) from the class $\left(L L^{-}, R R^{+}\right)$(negative deviation).

- Similarly, the study of $A_{L L}^{P V}\left(e^{+}\right)$distinguishes $\left(L R^{+}, R L^{-}\right)$from $\left(L R^{-}, R L^{+}\right)$. 
- Then the comparison of $A_{L L}^{P V}\left(e^{-}\right)$and $A_{L L}^{P V}\left(e^{+}\right)$allows to pin down the origin of the effect coming from one of these four classes : $\left(L L^{+}, R R^{-}\right)\left(L L^{-}, R R^{+}\right)\left(L R^{+}, R L^{-}\right)$ $\left(L R^{-}, R L^{+}\right)$.

The second step is to study the charge asymmetry $B_{2}^{2}$, which is represented on Figure 22. We consider this asymmetry because we have seen that it is the one which has the best discovery potential for $L R^{ \pm}$models (under the assumption $\Delta B_{\text {syst }} / B=10 \%$ ).

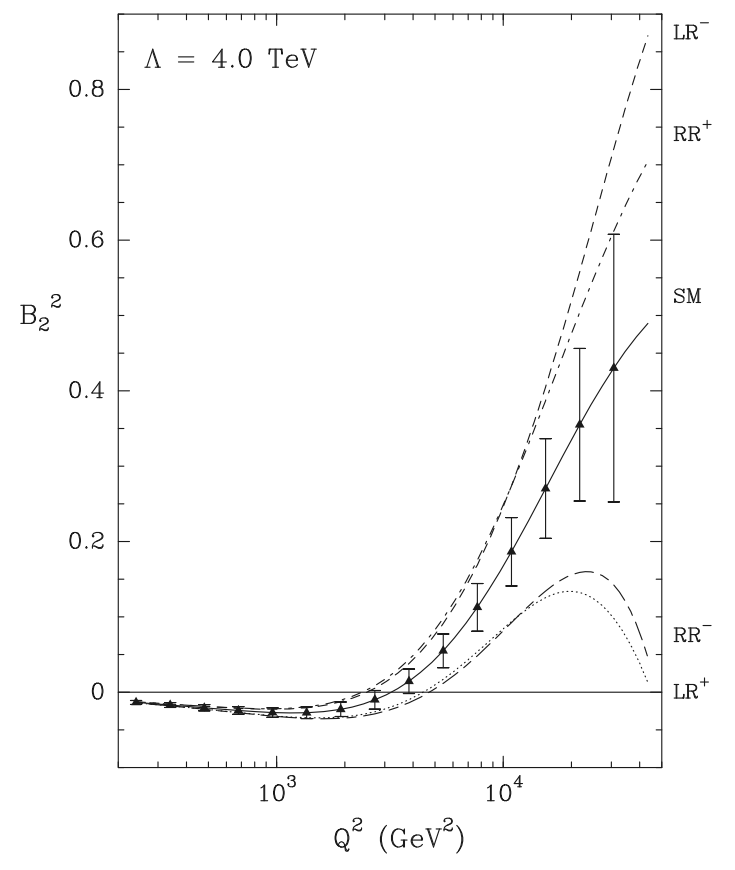

Figure 2: Same as Figure 1 for $B_{2}^{2}$.

Note that, as for $A_{L L}^{P V}\left(e^{ \pm}\right)$, we can also obtain an approximate formula for the numerator of $B_{2}^{2}$ :

$$
\operatorname{num}\left[B_{2}^{2}\right] \simeq-\epsilon K \eta(1-\eta \prime) u^{+}
$$

The properties of this charge asymmetry are the followings :

- An effect on $B_{2}^{2}$ indicates the class $\left(L R^{ \pm}, R R^{ \pm}\right)$. The direction of the deviation from SM expectations allows the distinction between $\left(R R^{+}, L R^{-}\right)$for a positive deviation, and $\left(R R^{-}, L R^{+}\right)$for a negative one.

- An absence of deviation on $B_{2}^{2}$ associated with a non-standard effect on an other observable $\left(A_{L L}^{P V}\left(e^{ \pm}\right)\right.$in particular $)$, reveals the presence of the class $\left(L L^{ \pm}, R L^{ \pm}\right)$.

The sum of the informations obtained from the three asymmetries $A_{L L}^{P V}\left(e^{-}\right), A_{L L}^{P V}\left(e^{+}\right)$and $B_{2}^{2}$, allows us to identify the chiral structure and the sign $\epsilon$ of the new interaction (for the 
individual models). This can be seen from Table 4.1:

\begin{tabular}{|c||c|c|c|c||c|c|c|c|}
\hline & $\eta_{L L}^{+}$ & $\eta_{R R}^{+}$ & $\eta_{L R}^{+}$ & $\eta_{R L}^{+}$ & $\eta_{L L}^{-}$ & $\eta_{R R}^{-}$ & $\eta_{L R}^{-}$ & $\eta_{R L}^{-}$ \\
\hline \hline$A_{L L}^{P V}\left(e^{-}\right)$ & + & - & 0 & 0 & - & + & 0 & 0 \\
\hline$A_{L L}^{P V}\left(e^{+}\right)$ & 0 & 0 & - & + & 0 & 0 & + & - \\
\hline$B_{2}^{2}$ & 0 & + & - & 0 & 0 & - & + & 0 \\
\hline
\end{tabular}

Table 4.1: "Deviation signatures" for the individual models.

In this table,,+- 0 correspond to positive, negative and "no-" deviation from the SM. We see that each model has a different "deviation signature", which indicates a clear identification of the chiral structure for these individual models. This identification could be realised up to a certain scale, the "identification limit" $\Lambda_{i d}$, which corresponds roughly to the lower discovery limit obtained from $A_{L L}^{P V}\left(e^{-}\right), A_{L L}^{P V}\left(e^{+}\right)$and $B_{2}^{2}$. We find (see Table 3.3) $\Lambda_{i d} \sim 5.1 \mathrm{TeV}$.

\subsection{Models with chiral structure involving several individual terms}

In this subsection we do not attempt to realize a general study of these models with a more complicated chiral structure than the individual models. Here, we just want to remark how the chiral structure of some models with an extended structure, like the ones advocated to explain the possible HERA anomaly, could be constrained at a general level.

First, we have to note that, when several chiralities are present simultaneously some cancellations may occur in the different asymmetries, which can reduce drastically their analysing and discovery potentials. However, in general, the three PV asymmetries $A_{L L}^{P V}\left(e^{-}\right), A_{L L}^{P V}\left(e^{+}\right)$and $B_{2}^{2}$ are sufficient to obtain some valuable informations on the chiral structure of the new interaction. This can be seen for the different models, advocated to explain the possible HERA anomaly, which are the following.

- Barger et al. 25 have defined two models : M1 : $\eta_{L R}^{\epsilon}=\eta_{R L}^{\epsilon}$ and $\mathrm{M} 2: \eta_{L R}^{\epsilon}=\eta_{R L}^{-\epsilon}$, the other $\eta_{i j}^{\epsilon}$ coefficients being set to zero.

- The authors of ref.[17] defined three models : VV : $\eta_{L L}^{\epsilon}=\eta_{R R}^{\epsilon}=\eta_{L R}^{\epsilon}=\eta_{R L}^{\epsilon}$, AA : $\eta_{L L}^{\epsilon}=\eta_{R R}^{\epsilon}=\eta_{L R}^{-\epsilon}=\eta_{R L}^{-\epsilon}$ and VA $: \eta_{L L}^{-\epsilon}=\eta_{R R}^{\epsilon}=\eta_{L R}^{\epsilon}=\eta_{R L}^{-\epsilon}$. (For completeness, we consider also the model AV : $\eta_{L L}^{-\epsilon}=\eta_{R R}^{\epsilon}=\eta_{L R}^{-\epsilon}=\eta_{R L}^{\epsilon}$ )

Roughly, when we add the contributions of the individual models for a given asymmetry, we find for M1, for instance, that we will have no effect for $A_{L L}^{P V}\left(e^{+}\right)$and a "normal" effect for $B_{2}^{2}$. We mean by "normal" effect, an effect of the same magnitude than the one induces by an individual model only. Similarly, we call a "double" effect, a deviation due to a certain model, which is twice larger than the one due to an individual model. 
For instance, for M2, we will have a double effect for $A_{L L}^{P V}\left(e^{+}\right)$and a normal effect for $B_{2}^{2}$ (see Figures 1-2). Analogously for the other models, we naively obtain the "deviation signatures" presented in Table 4.2.

\begin{tabular}{|c||c|c|c|c|c|c|}
\hline & M1 & M2 & VV & AA & VA & AV \\
\hline \hline$A_{L L}^{P V}\left(e^{-}\right)$ & 0 & 0 & 0 & 0 & $-2 \epsilon$ & $-2 \epsilon$ \\
\hline$A_{L L}^{P V}\left(e^{+}\right)$ & 0 & $-2 \epsilon$ & 0 & 0 & $-2 \epsilon$ & $2 \epsilon$ \\
\hline$B_{2}^{2}$ & $-\epsilon$ & $-\epsilon$ & 0 & $2 \epsilon$ & 0 & $2 \epsilon$ \\
\hline
\end{tabular}

Table 4.2: "Deviation signatures" for the models with several terms.

We find that, in general, the "deviation signatures" are distinct between these models and also distinct from the one's of the individual models given in Table 4.1. However, some ambiguities remain with several models. For instance, it could be difficult to distinguish between the M2 model and the $\eta_{L R}^{\epsilon}$ term alone. Moreover, the VV model does not give any deviation, since it conserves parity ? It is for such distinctions that the studies of the different PC spin asymmetries are needed.

Indeed (see appendix A), it appears that the cross sections defined with identical helicities (i.e. $\sigma_{t}^{\lambda \lambda}$ with $\lambda=\lambda_{e}=\lambda_{p}$ ) are mainly sensitive to one chirality only. Conversely, the cross sections $\sigma_{t}^{\lambda-\lambda}$ are almost unsensitive to new physics. Then if we construct some spin asymmetries defined with these two types of cross sections, we will obtain some asymmetries which are mainly sensitive to one chirality only. Such asymmetries correspond to the PC asymmetries defined in section 3 (eq.(11-12)). We recall that we have found that they are less sensitive to new physics than the PV asymmetries (see Table 3.3). These four PC asymmetries are represented for the individual models on Figure 3 .

In conclusion, we deduce from this rough analysis, that in general, the PV spin asymmetries are sufficient to obtain some crucial informations on the chiral structure of the new interaction, but it is necessary to study the PC asymmetries in order to disentangle some hidden effects.

\subsection{Remarks on the one-spin asymmetries}

The procedure described above could be easily adapted to the one-spin asymmetries. If we remember that the one-spin cross sections are given in terms of the two-spin cross sections, we see immediatly that the numerators of $A_{L L}^{P V}\left(e^{-}\right), A_{L L}^{P V}\left(e^{+}\right)$and $B_{2}^{2}$ are contained in the numerator of the one-spin asymmetries $A_{L}\left(e^{-}\right), A_{L}\left(e^{+}\right)$and $B_{1}^{2}$, respectively. Then, the study of these three one-spin asymmetries is roughly equivalent to that of the three two-spin asymmetries. However, several facts make this one-spin analysis less significant:

- The presence in the numerators of the $\sigma_{t}^{\lambda-\lambda}$ cross sections decreases the discovery potential, in the sense that the "informations" contained in the numerators are

${ }^{6}$ In fact, there are some small effects due to Z-CI interferences, sizeable in the high $Q^{2}$ domain, if $\Lambda$ is not too large. 

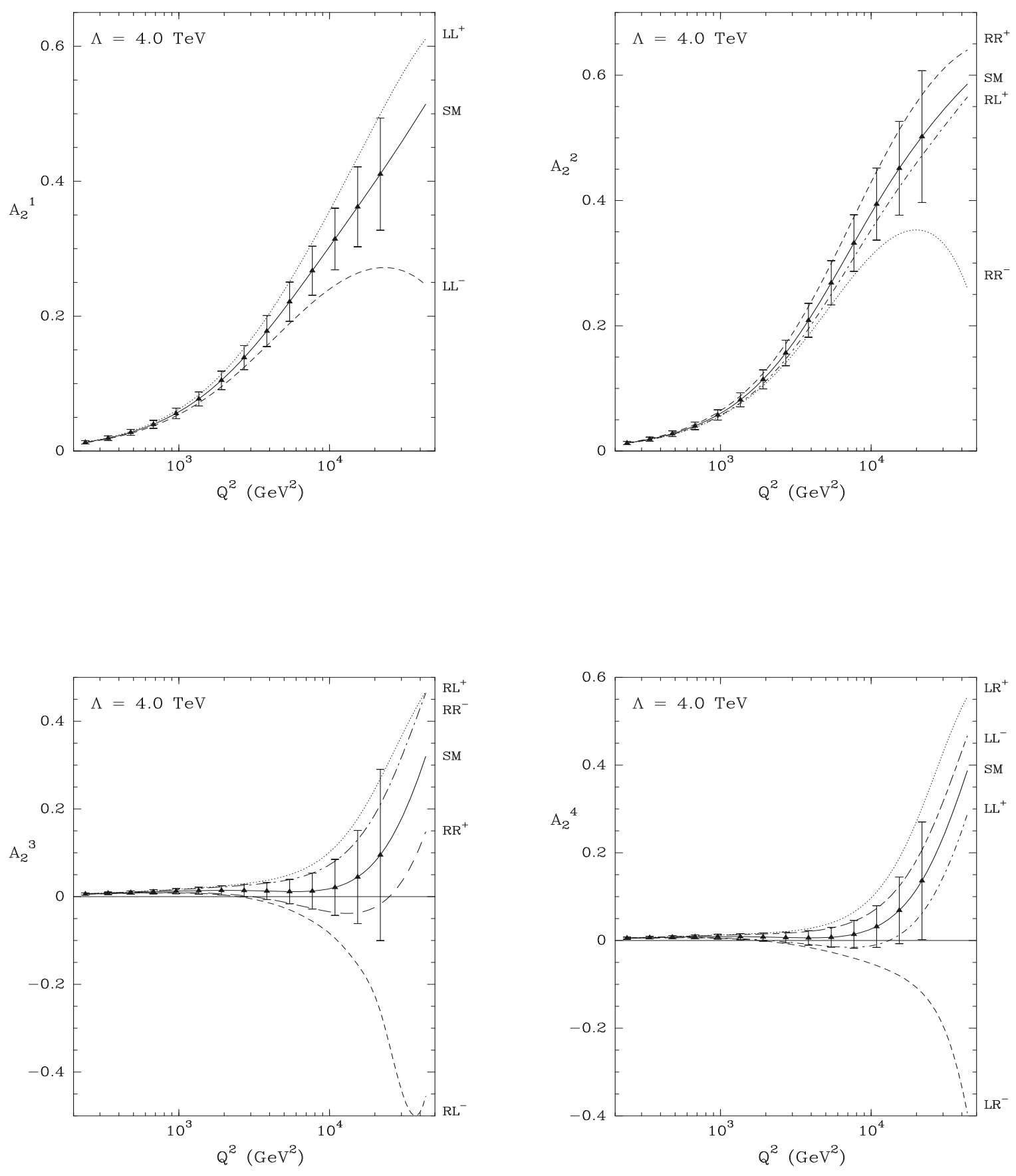

Figure 3: Same as Figure 1 for Parity Conserving Asymmetries. 
diluted, since these cross sections are weakly sensitive to new physics. This can be understood by the examination of the ratio of the dominant contributions of the one-spin to two-spin cross sections, given by (see appendix A) :

$$
\frac{\sigma_{t}^{\lambda_{e}}}{\sigma_{t}^{\lambda_{e} \lambda_{p}}} \simeq \frac{u / 2}{u^{+}}=1-\frac{\Delta u}{2 u^{+}}<1 \quad \text { since } \Delta u>0 .
$$

This is confirmed by the results presented in Tables 3.2 and 3.3, where we see that the one-spin asymmetries are sensitive to CI for some energy scales smaller by roughly $1 \mathrm{TeV}$ in comparison to the sensitivity of the two-spin asymmetries.

- We have at hand a smaller number of spin and charge asymmetries in comparison to the two-spin case. In particular, we cannot define the Parity Conserving asymmetries $A_{2}^{1-4}$. Then, if the chiral structure of the new interaction is complex, and if some cancellations occur between the different individual terms, we can lose some important informations on this chiral structure]!

\section{Conclusions}

- The HERA collider, with a high integrated luminosity $\left(L_{t o t}=1 f b^{-1}\right)$, could give some strong bounds on the energy scale of a possible new CI. For constructive interferences, the scale $\Lambda$ is of the order of $6 \mathrm{TeV}$, and for destructive interferences we get $\Lambda \sim 5 \mathrm{TeV}$. The availability of polarized leptons and protons beams will not increase significantly these bounds, except for destructive interferences. When only lepton polarization is available the sensitivity is strongly reduced, the limits decreasing by roughly $1 \mathrm{TeV}$.

- Contrarily to the analysis that can be performed for unpolarized collisions, the studies of several spin and charge asymmetries defined in a polarized context, can give some crucial informations on the chiral structure of the new interaction. The nature, constructive or destructive, of the interferences with photon exchange, can be also disentangled. It appears that the asymmetries defined for double polarized collisions are more well-suited for this chiral structure analysis, than the asymmetries defined with lepton polarization only. In any case, the availability of electron plus positron beams is mandatory, in order to cover all the possible chiralities.

- This analysis has assumed an universality for $u$ and $d$ quarks contributions $\left(\eta_{i j}^{u}=\right.$ $\eta_{i j}^{d}$ ). However, in a proton, the $u$-quark distribution is dominant. Consequently, the results presented here essentially constrain the presence of a new interaction in the electron-up-quark sector. To constrain an electron-down-quark new interaction, the

\footnotetext{
${ }^{7}$ One can argue that a fitting procedure to the data, combining unpolarized and single polarized observables, will constrain all the different chiralities 45. However, the arguments presented here, indicate that the fitting procedure in the one-spin case will be less sensitive to new physics than in the two-spin case, which will results in weaker constraints.
} 
isospin symmetry indicates that the protons have to be replaced by neutrons, so one should use electron- $H e^{3}$ collisions, an option which is also under consideration at HERA [15.

- In this paper we concentrate on the presence of some new CI. However, there are some equivalences between such CI and the exchange of some new Leptoquarks or $\not R_{p}$-squarks. It is well-known [24] that such bosons have to be chiral (i.e. with PV couplings) to evade the present experimental constraints. Then, the general results on the chiral structure analysis presented here, could be applied to these new bosons if they have an accessible mass at HERA, since, in this case, they will induce some important effects in the different PV spin asymmetries. These questions will be analyzed in a forthcoming paper [46].

\section{Acknowledgments}

I am indebted to P. Taxil and J. Soffer for invaluable discussions, and to A. De Roeck, H.-U. Martyn, B. Kamal, C. Benchouk, G. Bunce, M.J. Tannenbaum and J. Feltesse for very helpful informations and discussions. In particular, I am gratefull to J. Soffer, M.J. Tannenbaum and all the active members of the "RHIC Spin Discussion Team" at the RIKEN-BNL Research Center, for encouraging me to write the appendix B. I would like to thank M. Karliner who has initiated this work (a long time ago !). I wish to acknowledge Professors T.D. Lee and A. Baltz for their invitation at the RIKEN-BNL Research Center, where part of this work has been done. Finally, I thank P. Esposito for her kind hospitality at BNL. 


\section{A Formulas}

We present in this appendix, the set of formulas necessary to calculate the double polarized cross sections, the spin and charge asymmetries involved in the present analysis and, finally, the dominant contributions coming from the presence of a new CI.

\section{A.1 Cross sections}

\section{- $\underline{\text { Process }}$}

The single polarized cross sections are given in [7, 8]. Here we give the cross sections in the $(s, t, u)$ notations.

The collisions between charged leptons and protons, in the neutral current channel, correspond to the process : $\vec{e}^{ \pm} \vec{p} \longrightarrow e^{ \pm} X$, whose cross section is given by :

$$
\sigma_{t}^{\lambda_{e} \lambda_{p}} \equiv{\frac{d \sigma_{t}}{d Q^{2}}}^{\lambda_{e} \lambda_{p}}=\sum_{q} \int_{x_{\min }}^{x_{\max }} d x \sum_{\lambda_{q}}{\frac{d \hat{\sigma}_{t}}{d \hat{t}}}^{\lambda_{e} \lambda_{q}} q_{\lambda_{p}}^{\lambda_{q}}\left(x, Q^{2}\right)
$$

where $\lambda_{e}, \lambda_{p}$ and $\lambda_{q}$ are the helicities of the charged lepton, proton and parton (quark or antiquark), respectively. The label $t= \pm$ of $\sigma_{t}$ corresponds to the electric charge of the colliding lepton. $\sum_{q}$ represent the sum over all the quark and antiquark flavors present inside the proton. Concerning the kinematical variables, let $P_{N}, P_{l}$ and $P_{l^{\prime}}$ be the momentum of the proton, the incoming lepton and the scattered lepton. If $P_{q}=x P_{N}$ is the momentum of the parton carrying the momentum fraction $x$ of the nucleon, the subprocess variables $\hat{s}, \hat{t}$ and $\hat{u}$ are given by :

$$
\begin{aligned}
& \hat{s}=\left(P_{q}+P_{l}\right)^{2} \simeq+2 P_{q} P_{l}=x 2 P_{N} P_{l}=x s \\
& \hat{t}=\left(P_{l}-P_{l^{\prime}}\right)^{2}=t=-Q^{2} \\
& \hat{u}=\left(P_{q}-P_{l^{\prime}}\right)^{2} \simeq-2 P_{q} P_{l^{\prime}}=x u=-x(1-y) s,
\end{aligned}
$$

where the usual variable $y$ is defined by $y=P_{N}\left(P_{l}-P_{l^{\prime}}\right) / P_{N} P_{l}=Q^{2} / x s$. The boundary condition $x \leq 1$ implies $y \geq Q^{2} / s$. However, experimentally the $y$ variable is constrained in the range [28]: $y_{\min }^{e x p}=0.01$ and $y_{\max }^{\exp }=0.8$, but one is hoping to reach in the future 29]: $y_{\max }^{\exp }=0.95$. The integration limits are : $x_{\min }=Q^{2} / s y_{\max }, x_{\max }=Q^{2} / s y_{\min }$ with $y_{\min }=\max \left(y_{\min }^{\text {exp }}, Q^{2} / s\right), y_{\max }=y_{\max }^{\text {exp }}$. In the high $Q^{2}$ region we have roughly : $x_{\text {min }} \simeq Q^{2} / s, x_{\max } \simeq 1$.

We denote by $q_{\lambda_{p}}^{\lambda_{q}}\left(x, Q^{2}\right)$ the parton distribution for the parton $q$ inside a proton of helicity $\lambda_{p}$, with momentum fraction $x$ and helicity $\lambda_{q}$, at the energy scale $Q^{2}$. These distributions are related to the parallel and anti-parallel distributions by : $q_{+}=q_{+}^{+}=q_{-}^{-}, q_{-}=$ $q_{+}^{-}=q_{-}^{+}$, which are related to the usual unpolarized and polarized parton distributions 
by : $q=q_{+}+q_{-}$and $\Delta q=q_{+}-q_{-}$.

\section{- Subprocesses}

Using the notations of [47, the cross section of the elementary subprocess $\vec{e} \vec{q} \rightarrow e q$ is given by :

$$
{\frac{d \hat{\sigma}_{t}}{d \hat{t}}}^{\lambda_{e} \lambda_{q}}=\frac{\pi}{\hat{s}^{2}} \sum_{\alpha, \beta} T_{\alpha, \beta}^{\lambda_{e} \lambda_{q}}\left(e^{t}, q\right)
$$

where $T_{\alpha, \beta}^{\lambda_{e} \lambda_{q}}\left(e^{t}, q\right)$ is the square matrix element for $\alpha$ and $\beta$ boson exchange, or the exchange process replaced by a CI; $q$ being a quark or an antiquark. The $T_{\alpha, \beta}^{\lambda_{e} \lambda_{q}}\left(e^{t}, q\right)$ are given below for $\alpha, \beta=\gamma, Z, C I$. We have omitted the hat symbol of the variables $\hat{s}, \hat{t}$ and $\hat{u}$, for clarity.

Subprocess $\quad e^{-} q \longrightarrow e^{-} q$ :

$$
\begin{aligned}
& T_{\gamma \gamma}=2 e_{q}^{2} \frac{\alpha^{2}}{t^{2}}\left[\left(1+\lambda_{e} \lambda_{q}\right) s^{2}+\left(1-\lambda_{e} \lambda_{q}\right) u^{2}\right], \\
& T_{Z Z}=\frac{\alpha_{Z}^{2}}{t_{Z}^{2}}\left[\left(C_{e L}^{2} C_{q L}^{2}\left(1-\lambda_{e}\right)\left(1-\lambda_{q}\right)+C_{e R}^{2} C_{q R}^{2}\left(1+\lambda_{e}\right)\left(1+\lambda_{q}\right)\right) s^{2}\right. \\
& \left.+\left(C_{e L}^{2} C_{q R}^{2}\left(1-\lambda_{e}\right)\left(1+\lambda_{q}\right)+C_{e R}^{2} C_{q L}^{2}\left(1+\lambda_{e}\right)\left(1-\lambda_{q}\right)\right) u^{2}\right], \\
& T_{\gamma Z}=-2 e_{q} \frac{\alpha \alpha_{Z}}{t t_{Z}}\left[\left(C_{e L} C_{q L}\left(1-\lambda_{e}\right)\left(1-\lambda_{q}\right)+C_{e R} C_{q R}\left(1+\lambda_{e}\right)\left(1+\lambda_{q}\right)\right) s^{2}\right. \\
& \left.+\left(C_{e L} C_{q R}\left(1-\lambda_{e}\right)\left(1+\lambda_{q}\right)+C_{e R} C_{q L}\left(1+\lambda_{e}\right)\left(1-\lambda_{q}\right)\right) u^{2}\right], \\
& T_{C I C I}=\frac{1}{2 \Lambda^{4}}\left[\left(\left(1+\eta \eta^{\prime}\right)\left(1+\lambda_{e} \lambda_{q}\right)+\left(-\eta-\eta^{\prime}\right)\left(\lambda_{e}+\lambda_{q}\right)\right) s^{2}\right. \\
& \left.+\left(\left(1-\eta \eta^{\prime}\right)\left(1-\lambda_{e} \lambda_{q}\right)+\left(\eta-\eta^{\prime}\right)\left(-\lambda_{e}+\lambda_{q}\right)\right) u^{2}\right], \\
& T_{\gamma C I}=-\epsilon e_{q} \frac{\alpha}{t \Lambda^{2}}\left[\left(\left(1+\eta \eta^{\prime}\right)\left(1+\lambda_{e} \lambda_{q}\right)+\left(-\eta-\eta^{\prime}\right)\left(\lambda_{e}+\lambda_{q}\right)\right) s^{2}\right. \\
& \left.+\left(\left(1-\eta \eta^{\prime}\right)\left(1-\lambda_{e} \lambda_{q}\right)+\left(\eta-\eta^{\prime}\right)\left(-\lambda_{e}+\lambda_{q}\right)\right) u^{2}\right] \text {, } \\
& T_{Z C I}=\epsilon \frac{\alpha_{Z}}{2 t_{Z} \Lambda^{2}}\left[\left(C_{e L} C_{q L}(1+\eta)\left(1+\eta^{\prime}\right)\left(1-\lambda_{e}\right)\left(1-\lambda_{q}\right)\right.\right. \\
& \left.+C_{e R} C_{q R}(1-\eta)\left(1-\eta^{\prime}\right)\left(1+\lambda_{e}\right)\left(1+\lambda_{q}\right)\right) s^{2} \\
& +\left(C_{e L} C_{q R}(1+\eta)\left(1-\eta^{\prime}\right)\left(1-\lambda_{e}\right)\left(1+\lambda_{q}\right)\right. \\
& \left.\left.+C_{e R} C_{q L}(1-\eta)\left(1+\eta^{\prime}\right)\left(1+\lambda_{e}\right)\left(1-\lambda_{q}\right)\right) u^{2}\right] \text {, }
\end{aligned}
$$

where $\alpha$ is the electromagnetic coupling, $\alpha_{Z}=\alpha / \sin ^{2} \theta_{W} \cos ^{2} \theta_{W}, t_{Z}=t-M_{Z}^{2} \cdot C_{f L}$ and $C_{f R}$ are the usual Left-handed and Right-handed couplings of the $Z$ to the fermion 
$f$, given by $C_{f L}=I_{3}^{f}-e_{f} \sin ^{2} \theta_{W}, C_{f R}=-e_{f} \sin ^{2} \theta_{W}$ with $I_{3}^{f}= \pm 1 / 2$. The parameters $\eta$ and $\eta^{\prime}$ characterize the chiral structure of the CI with the conventions : $L L(\eta=+1, \eta \prime=+1), R R(-1,-1), L R(+1,-1), R L(-1,+1)$.

Subprocess $\quad e^{+} q \longrightarrow e^{+} q:$

The squared matrix elements $T_{\alpha, \beta}$ are obtained from the six preceding equations with the following changes : $s \longleftrightarrow u$ and $\lambda_{e} \longleftrightarrow-\lambda_{e}$ (equivalent to $C_{e L} \longleftrightarrow C_{e R}$ and $\eta \longleftrightarrow-\eta)$

Subprocess $\quad e^{ \pm} \bar{q} \longrightarrow e^{ \pm} \bar{q}:$

The $T_{\alpha, \beta}$ are obtained from the ones for $e^{ \pm} q$ scattering after the transformations : $s \longleftrightarrow u$ and $\lambda_{q} \longleftrightarrow-\lambda_{q}$.

The dominant term being $T_{\gamma C I}$ (see below), $\epsilon=+1$ corresponds to constructive interferences since $t<0$ and that in a proton the $u$-quark, with $e_{u}>0$, is dominant.

\section{A.2 Dominant terms}

Some general arguments on the dominant effects, due to the presence of a CI, will allow us to obtain some simple relations for the cross sections, which in turn will give the possibility to predict the behavior of the different asymmetries. For simplicity, we realize these studies with the double differential cross sections $d \sigma^{\lambda_{e} \lambda_{p}} / d x d Q^{2}$, the integration over $x$ does not change the results.

The important point is that we study the events with the highest energies, namely with some high $Q^{2}, x$ and $y$. Then we can make the following reasonable hypothesis :

- $H 1 \rightarrow \hat{s}^{2} \gg \hat{u}^{2}$

- $H 2 \rightarrow u\left(x, Q^{2}\right)>d\left(x, Q^{2}\right)$,

- $H 3 \rightarrow \Delta u\left(x, Q^{2}\right)>0$ (and increasing with $x$, for high $x$ ),

- $H 4 \rightarrow \bar{q}\left(x, Q^{2}\right) \ll q\left(x, Q^{2}\right)$.

With $H 4$ the process cross section corresponding to eq.(A.1) becomes:

$$
\sigma_{t}^{\lambda_{e} \lambda_{p}} \equiv \frac{d \sigma_{t}^{\lambda_{e} \lambda_{p}}}{d x d Q^{2}}=\sum_{q} \frac{d \hat{\sigma}_{t}{ }^{\lambda_{e} \lambda_{p}}}{d \hat{t}} q^{+}\left(x, Q^{2}\right)+{\frac{d \hat{\sigma}_{t}^{\lambda_{e}-\lambda_{p}}}{d \hat{t}}}^{-} q^{-}\left(x, Q^{2}\right) .
$$

With $H 2$ we get $\left(\hat{\sigma}_{t}^{\lambda_{e} \lambda_{p}} \equiv d \hat{\sigma}_{t}^{\lambda_{e} \lambda_{p}} / d \hat{t}\right)$ :

$$
\sigma_{t}^{\lambda_{e} \lambda_{p}} \simeq \hat{\sigma}_{t}^{\lambda_{e} \lambda_{p}} u^{+}\left(x, Q^{2}\right)+\hat{\sigma}_{t}^{\lambda_{e}-\lambda_{p}} u^{-}\left(x, Q^{2}\right) .
$$


For high values of $\Lambda$, the squared amplitudes for the CI $\left(\sim 1 / \Lambda^{4}\right)$ are suppressed compared to the interferences $\gamma C I\left(\sim 1 / Q^{2} \Lambda^{2}\right)$ or $Z C I\left(\sim 1 /\left(Q^{2}+M_{Z}^{2}\right) \Lambda^{2}\right)$. In the following, for simplicity, we will neglect the interference term $Z C I$ which is not dominant.

Then, we assume that the dominant terms for the CI come from the interferences $\gamma C I$, given by eq.(A.10). In terms of the helicities, using $H 1$, we deduce that the dominant matrix elements squared are :

$$
\begin{aligned}
& T_{\gamma C I}^{--}\left(e^{-}\right)=\epsilon \frac{K}{2 \pi} \hat{s}^{2}(1+\eta)\left(1+\eta^{\prime}\right), \\
& T_{\gamma C I}^{++}\left(e^{-}\right)=\epsilon \frac{K}{2 \pi} \hat{s}^{2}(1-\eta)\left(1-\eta^{\prime}\right), \\
& T_{\gamma C I}^{--}\left(e^{+}\right)=\epsilon \frac{K}{2 \pi} \hat{s}^{2}(1-\eta)\left(1+\eta^{\prime}\right), \\
& T_{\gamma C I}^{++}\left(e^{+}\right)=\epsilon \frac{K}{2 \pi} \hat{s}^{2}(1+\eta)\left(1-\eta^{\prime}\right),
\end{aligned}
$$

where $K=8 \pi \alpha / 3 \Lambda^{2} Q^{2}$. We find that the dominant cross sections for the subprocesses are of the form $\hat{\sigma}^{\lambda \lambda}\left(\lambda=\lambda_{e}=\lambda_{q}\right)$. We deduce that the dominant cross sections for the process are :

$$
\sigma_{t}^{\lambda \lambda} \simeq \hat{\sigma}_{t}^{\lambda \lambda} u^{+} \quad \text { and } \quad \sigma_{t}^{\lambda-\lambda} \simeq \hat{\sigma}_{t}^{\lambda \lambda} u^{-} .
$$

The hypothesis $H 3$ indicates that the dominant process cross sections are of the form $\sigma_{t}{ }^{\lambda \lambda}$ (and not $\sigma_{t}^{\lambda-\lambda}$ ), and they correspond to :

$$
\sigma_{t}^{\lambda \lambda} \simeq \hat{\sigma}_{t}^{\lambda \lambda} u^{+}=\frac{\pi}{\hat{s}^{2}} T_{\gamma C I}^{\lambda \lambda} u^{+}
$$

We conclude that $\sigma_{-}^{--}$is sensitive to $L L^{ \pm}, \sigma_{-}^{++}$to $R R^{ \pm}, \sigma_{+}^{--}$to $R L^{ \pm}$and $\sigma_{+}^{++}$to $L R^{ \pm}$. An immediate consequence is that the unpolarized cross sections $\sigma_{-}$and $\sigma_{+}$are sensitive to $\left(L L^{ \pm}, R R^{ \pm}\right)$and $\left(L R^{ \pm}, R L^{ \pm}\right)$, respectively.

For the one-spin cross sections we get :

$$
\sigma_{t}^{\lambda}=\frac{1}{2}\left(\sigma_{t}^{\lambda \lambda}+\sigma_{t}^{\lambda-\lambda}\right) \simeq \frac{1}{2} \hat{\sigma}_{t}^{\lambda \lambda} u
$$

The sensitivity to each chirality is trivial. Moreover, the comparison of this equation with eq.A.15), indicates that the two-spin cross sections are more sensitive to the CI than the one-spin ones, because :

$$
\frac{\sigma_{t}^{\lambda}}{\sigma_{t}^{\lambda \lambda}} \simeq \frac{u / 2}{u^{+}}=1-\frac{\Delta u}{2 u^{+}}<1 \quad \text { since } \Delta u>0
$$

The behavior of the different asymmetries is easily obtainable from the above equations, because it is governed by its numerator, since the denominators are dominated by the SM. For instance, we obtain for the asymmetries considered in the text :

$$
\operatorname{num}\left[A_{L L}^{P V}\left(e^{-}\right)\right] \simeq \epsilon K(+\eta+\eta \prime) u^{+}
$$




$$
\begin{gathered}
\operatorname{num}\left[A_{L L}^{P V}\left(e^{+}\right)\right] \simeq \epsilon K(-\eta+\eta \prime) u^{+}, \\
\operatorname{num}\left[B_{2}^{2}\right] \simeq-\epsilon K \eta(1-\eta \prime) u^{+},
\end{gathered}
$$

and for the PC asymmetries (see eq.(A.15)) :

$$
\begin{aligned}
\operatorname{num}\left[A_{2}^{1-4}\right] & =\sigma_{t}^{\lambda \lambda}-\sigma_{t}^{\lambda-\lambda} \simeq \hat{\sigma}_{t}^{\lambda \lambda} \Delta u \\
\operatorname{num}\left[A_{2}^{1}\right] & \simeq \epsilon \frac{K}{2}(1+\eta)\left(1+\eta^{\prime}\right) \Delta u \\
\operatorname{num}\left[A_{2}^{2}\right] & \simeq \epsilon \frac{K}{2}(1-\eta)\left(1-\eta^{\prime}\right) \Delta u \\
\operatorname{num}\left[A_{2}^{3}\right] & \simeq \epsilon \frac{K}{2}(1-\eta)\left(1+\eta^{\prime}\right) \Delta u \\
\operatorname{num}\left[A_{2}^{4}\right] & \simeq \epsilon \frac{K}{2}(1+\eta)\left(1-\eta^{\prime}\right) \Delta u
\end{aligned}
$$

Then, $A_{L L}^{P V}\left(e^{-}\right)$is sensitive to $\left(L L^{ \pm}, R R^{ \pm}\right), A_{L L}^{P V}\left(e^{+}\right)$to $\left(L R^{ \pm}, R L^{ \pm}\right), B_{2}^{2}$ to $\left(R R^{ \pm}, L R^{ \pm}\right)$, $A_{2}^{1}$ to $L L^{ \pm}, A_{2}^{2}$ to $R R^{ \pm}, A_{2}^{3}$ to $R L^{ \pm}$and $A_{2}^{4}$ to $L R^{ \pm}$.

\section{B "Experimental asymmetries"/definition of $\Delta A_{\text {stat }}$}

Experimentally the particle beams are never fully polarized, so we need to introduce some degree of polarization $P$. Then, there is a shift between the values of the observable defined theoretically $(P=1)$ and the one measured experimentally $(P \neq 1)$.

Only in the two cases $A_{L}$ and $A_{L L}^{P C}$, the relation between the two asymmetries ("theoretical" and "experimental") is simple, and a suitable redefinition of the measured asymmetry makes the situation clear (see below). In some other cases, like the double PC spin asymmetry $A_{\|}$measured in polarized deep inelastic scattering at low $Q^{2}$, some relevant assumptions (parity conservation) allow to obtain a similar simple relation (see below). However, in general, we do not have such simple relations between the two asymmetries, so we need a careful treatment, in order to take into account the degrees of polarization, and to define the statistical error of the asymmetries correctly.

The goal of this appendix is to show the procedure that we have used in the present article. This can be used in polarized hadronic collisions as well. Since it is relatively

\footnotetext{
${ }^{8} A_{L L}^{P C}$ is defined by $A_{L L}^{P C} \equiv A_{L L}=\left(\sigma^{+}+-\sigma^{+-}+\sigma^{--}-\sigma^{-+}\right) /\left(\sigma^{++}+\sigma^{+-}+\sigma^{--}+\sigma^{-+}\right)$
} 
technical, we introduce our notations, then, we illustrate our general procedure on $A_{L L}^{P V}$, since it is valid for any spin asymmetries. Finally, we give two examples which reproduce the simple relations mentioned above.

\section{B.1 Notations}

We note $\sigma^{\lambda_{a} \lambda_{b}}$ (三 $\left.\frac{d \sigma^{\lambda a \lambda_{b}}}{d X d Y_{\ldots}}\right)$ the cross section corresponding to the process $\vec{a}\left(\lambda_{a}\right) \vec{b}\left(\lambda_{b}\right) \rightarrow X$. The basic observables are : $\sigma^{++}, \sigma^{+-}, \sigma^{-+}$and $\sigma^{--}$. They are related to the one-spin cross sections by $\sigma^{\lambda_{a} 0}=\frac{1}{2}\left(\sigma^{\lambda_{a} \lambda_{b}}+\sigma^{\lambda_{a}-\lambda_{b}}\right)$, when only $\vec{a}$ are polarized, and similarly $\sigma^{0 \lambda_{b}}=\frac{1}{2}\left(\sigma^{\lambda_{a} \lambda_{b}}+\sigma^{-\lambda_{a} \lambda_{b}}\right)$, when only $\vec{b}$ are polarized. The unpolarized cross section is :

$$
\sigma^{00}=\frac{1}{4}\left(\sigma^{--}+\sigma^{++}+\sigma^{-+}+\sigma^{+-}\right)=\frac{1}{2}\left(\sigma^{-0}+\sigma^{+0}\right)=\frac{1}{2}\left(\sigma^{0-}+\sigma^{0+}\right) .
$$

Concerning the numbers of events, for a particular spin configuration $\left(\lambda_{a}, \lambda_{b}\right)$, we define $N^{\lambda_{a} \lambda_{b}}=L^{\lambda_{a} \lambda_{b}} \times \sigma^{\lambda_{a} \lambda_{b}}$. We make the special choice : $L^{\lambda_{a} \lambda_{b}}=\frac{1}{2} L^{\lambda_{a} 0}=\frac{1}{2} L^{0 \lambda_{b}}=\frac{1}{4} L^{00}$, where $L^{00}$ is the "total" integrated luminosity. Then we have :

$$
\begin{gathered}
N^{\lambda_{a} 0}=N^{\lambda_{a} \lambda_{b}}+N^{\lambda_{a}-\lambda_{b}} \quad, \quad N^{0 \lambda_{b}}=N^{\lambda_{a} \lambda_{b}}+N^{-\lambda_{a} \lambda_{b}}, \\
N^{00}=N^{--}+N^{++}+N^{-+}+N^{+-}=N^{-0}+N^{+0}=N^{0-}+N^{0+} .
\end{gathered}
$$

Now, if we define an asymmetry by :

$$
A=\frac{N_{1}-N_{2}}{N_{1}+N_{2}}
$$

its statistical error is 42]:

$$
\Delta A=\sqrt{\frac{1-A^{2}}{N_{1}+N_{2}}}=\frac{2}{\left(N_{1}+N_{2}\right)^{2}} \sqrt{N_{1} N_{2}\left(N_{1}+N_{2}\right)}=\frac{1-A^{2}}{\sqrt{1-A}} \frac{1}{\sqrt{2 N_{1}}}
$$

\section{B.2 General procedure : example on $A_{L L}^{P V}$}

Consider the process $\vec{a} \vec{b} \longrightarrow X$. Experimentally, we have a fraction $P_{a}$ of particles $a$ with helicity $\lambda_{a}$, and a fraction $1-P_{a}$ of unpolarized $a$ particles, which collide with a fraction $P_{b}$ of particles $b$ with helicity $\lambda_{b}$, and a fraction $1-P_{b}$ of unpolarized $b$ particles. We obtain the "experimental" cross section :

$$
\begin{aligned}
\sigma_{e x p}^{\lambda_{a} \lambda_{b}}= & P_{a} P_{b} \sigma^{\lambda_{a} \lambda_{b}}+P_{a}\left(1-P_{b}\right) \sigma^{\lambda_{a} 0}+\left(1-P_{a}\right) P_{b} \sigma^{0 \lambda_{b}}+\left(1-P_{a}\right)\left(1-P_{b}\right) \sigma^{00}( \\
= & \frac{1}{4}\left(1+P_{a}\right)\left(1+P_{b}\right) \sigma^{\lambda_{a} \lambda_{b}}+\frac{1}{4}\left(1-P_{a}\right)\left(1-P_{b}\right) \sigma^{-\lambda_{a}-\lambda_{b}} \\
& +\frac{1}{4}\left(1+P_{a}\right)\left(1-P_{b}\right) \sigma^{\lambda_{a}-\lambda_{b}}+\frac{1}{4}\left(1-P_{a}\right)\left(1+P_{b}\right) \sigma^{-\lambda_{a} \lambda_{b}}
\end{aligned}
$$


In terms of events, replace in the preceding equation (B.7), $\sigma$ by $N$.

We mean by "experimental" that the observable $\left(\mathcal{O}_{\exp }\right.$ with $\left.\mathcal{O} \equiv \sigma, A, B \ldots\right)$ is directly dependent on the degrees of polarization. In fact, $\mathcal{O}_{\exp }$ could correspond, on the one hand, to a theoretical quantity which takes into account the expected experimental conditions, or on the other hand, to a measured quantity in some actual experimental conditions $\left(\mathcal{O}_{\text {exp }} \equiv \mathcal{O}_{\text {meas }}\right)$. The former case is involved in any phenomenological simulation which intend to take into account the degrees of polarization, and we have to use eq.(B.7) to define the statistical error of the theoretical asymmetry properly. In the latter case, in order to reconstruct the number of events which are independent on the degrees of polarization, from the measured number of events, we will have to use the inverse formula of eq.(B.7) :

$$
\begin{aligned}
\sigma^{\lambda_{a} \lambda_{b}} & =\frac{1}{P_{a}^{\text {meas }} P_{b}^{\text {meas }}}\left[\frac{1}{4}\left(1+P_{a}^{\text {meas }}\right)\left(1+P_{b}^{\text {meas }}\right) \sigma_{\text {meas }}^{\lambda_{a} \lambda_{b}}+\frac{1}{4}\left(1-P_{a}^{\text {meas }}\right)\left(1-P_{b}^{\text {meas }}\right) \sigma_{\text {meas }}^{-\lambda_{a}-\lambda_{b}}\right. \\
- & \left.\frac{1}{4}\left(1+P_{a}^{\text {meas }}\right)\left(1-P_{b}^{\text {meas }}\right) \sigma_{\text {meas }}^{\lambda_{a}-\lambda_{b}}-\frac{1}{4}\left(1-P_{a}^{\text {meas }}\right)\left(1+P_{b}^{\text {meas }}\right) \sigma_{\text {meas }}^{-\lambda_{a} \lambda_{b}}\right]
\end{aligned}
$$

We insist on the fact that, now, $\sigma^{\lambda_{a} \lambda_{b}}$ is not a cross section given by theory, but a cross section constructed from the measured cross sections to be $P_{a}, P_{b}$ independent.

- Example on $A_{L L}^{P V}$

We begin this example in the framework of phenomenological simulations. In this case, $P_{a}$ and $P_{b}$ are the expected degrees of polarization for the beams $a$ and $b . A_{L L}^{P V}$ and $N^{--}, N^{++}, N^{-+}$and $N^{+-}$are some quantities predicted by theory, so, they are $P_{a}, P_{b}$ independent. We call $A_{L L}^{P V}($ exp $)$ and $N_{e x p}^{--}, N_{e x p}^{++}, N_{\text {exp }}^{-+}$and $N_{\text {exp }}^{+-}$some theoretical quantities which are $P_{a}, P_{b}$ dependent.

We call "theory 1 " the strategy which consists to use as the basic observable $A_{L L}^{P V}(\exp )$. In this case, with eq.(B.7), we have :

$$
\begin{aligned}
& A_{L L}^{P V}(\exp )=\frac{N_{e x p}^{--}-N_{e x p}^{++}}{N_{e x p}^{--}+N_{e x p}^{++}} \\
& \quad=\frac{\frac{1}{2}\left(P_{a}+P_{b}\right)\left[N^{--}-N^{++}\right]+\frac{1}{2}\left(P_{a}-P_{b}\right)\left[N^{-+}-N^{+-}\right]}{\frac{1}{2}\left[N^{--}+N^{++}+N^{-+}+N^{+-}\right]+\frac{1}{2} P_{a} P_{b}\left[N^{--}+N^{++}-N^{-+}-N^{+-}\right]}
\end{aligned}
$$

From this equation, we see that $A_{L L}^{P V}$ (exp) is not simply related to $A_{L L}^{P V}$. It means that, even if $A_{L L}^{P V}$ (exp) is defined with the ("experimental") number of events for two spin configurations, the presence of the degrees of polarization induces an admixture of the (theoretical) number of events for the four spin configurations.

However, this asymmetry is well-defined and its statistical error too :

$$
\Delta A_{L L}^{P V}(\exp )=\sqrt{\frac{1-\left(A_{L L}^{P V}(\exp )\right)^{2}}{N_{e x p}^{--}+N_{e x p}^{++}}} .
$$


Now, we call "theory 2" the strategy which consists to use as the basic observable $A_{L L}^{P V}$, which is simply defined by :

$$
A_{L L}^{P V}=\frac{N^{--}-N^{++}}{N^{--}+N^{++}} .
$$

Actually, as in ref. 43] for the study of $A_{L}$, we want $A_{L L}^{P V}$ to be the basic observable of the analysis. So the question arise : what is its statistical error? The answer boils into the fact that we want the two methods, theory 1 and theory 2 , to be equivalent, or practically, to have the same analyzing power $\left(i . e\right.$. the same $\left.\chi^{2} \sim(A / \Delta A)^{2}\right)$. Namely, we require that :

$$
\frac{A}{\Delta A}=\frac{A^{\exp }}{\Delta A^{\exp }}
$$

So, we define the statistical error of the theoretical asymmetry in the following way :

$$
\Delta A_{L L}^{P V}=\frac{A_{L L}^{P V}}{A_{L L}^{P V}(\exp )} \cdot \Delta A_{L L}^{P V}(\exp ) .
$$

Now, if we are in the framework of some experimental measurements, we have at hand some measured quantities : $P_{a}^{\text {meas }}, P_{b}^{\text {meas }}, N_{\text {meas }}^{--}, N_{\text {meas }}^{++}, N_{\text {meas }}^{-+}$and $N_{\text {meas }}^{+-}$. From these ones, we can construct (using eq.(B.8)) the $P_{a}, P_{b}$ independent number of events $N^{--}$, $N^{++}, N^{-+}$and $N^{+-}$.

Then, we call "measure 1" the strategy which consists to define as the basic observable an asymmetry which is dependent on the degrees of polarization. In this case, we have simply :

$$
\begin{gathered}
\left(A_{L L}^{P V}(\text { exp })\right)_{\text {meas }}=\frac{N_{\text {meas }}^{--}-N_{\text {meas }}^{++}}{N_{\text {meas }}^{--}+N_{\text {meas }}^{++}} \\
\left(\Delta A_{L L}^{P V}(\exp )\right)_{\text {meas }}=\sqrt{\frac{1-\left(A_{L L}^{P V}(\text { exp })\right)_{\text {meas }}^{2}}{N_{\text {meas }}^{--}+N_{\text {meas }}^{++}}}
\end{gathered}
$$

We have to compare this asymmetry to the one defined in "theory 1 ".

We call "measure 2" the strategy which consists to define as the basic observable an asymmetry which is independent on the degrees of polarization. Then, we construct $A_{L L}^{P V}$ by the use of eq.(B.8), and we find :

$$
\begin{aligned}
A_{L L}^{P V} & =\frac{\frac{1}{2 P_{a}^{\text {meas }}}\left[N_{\text {meas }}^{--}+N_{\text {meas }}^{-+}-N_{\text {meas }}^{+-}-N_{\text {meas }}^{++}\right]+\frac{1}{2 P_{b}^{\text {meas }}}\left[N_{\text {meas }}^{--}-N_{\text {meas }}^{-+}+N_{\text {meas }}^{+-}-N_{\text {meas }}^{++}\right]}{\frac{1}{2}\left[N_{\text {meas }}^{--}+N_{\text {meas }}^{-+}+N_{\text {meas }}^{+-}+N_{\text {meas }}^{++}\right]+\frac{1}{2 P_{a}^{\text {meas }} P_{b}^{\text {meas }}}\left[N_{\text {meas }}^{--}-N_{\text {meas }}^{-+}-N_{\text {meas }}^{+-}+N_{\text {meas }}^{++}\right]} \\
& =\frac{\frac{1}{2 P_{a}^{\text {meas }}}\left[N_{\text {meas }}^{-0}-N_{\text {meas }}^{+0}\right]+\frac{1}{2 P_{b}^{\text {meas }}}\left[N_{\text {meas }}^{0-}-N_{\text {meas }}^{0+}\right]}{\frac{1}{2} N_{\text {meas }}^{00}\left(1+\frac{1}{P_{a}^{\text {meas }} P_{b}^{\text {meas }}} A_{L L}^{\text {meas }}\right)}
\end{aligned}
$$


The associated statistical error is :

$$
\Delta A_{L L}^{P V}=\frac{A_{L L}^{P V}}{\left(A_{L L}^{P V}(\exp )\right)_{\text {meas }}} \cdot\left(\Delta A_{L L}^{P V}(\text { exp })\right)_{\text {meas }}
$$

We have to compare this asymmetry to the one defined in "theory 2 ".

The two procedures, "experimental" (i.e. theory/measure $1: P$ dependent) and "theoretical" (i.e. theory/measure 2: $P$ independent), are physically equivalent. This remark is valid for phenomenological simulations and for experimental measurements. We can choose the presentation of the results that we prefer. In order to present some curves which are independent of the assumed degrees of polarization, we have to use the strategy that we have called "theoretical". We have followed this procedure in this paper.

The strategies presented for $A_{L L}^{P V}$ are valid for any spin asymmetries.

Remark on the $A_{L L}^{P V}$ case :

From the equation (B.10), we have seen that there is no simple relation between $A_{L L}^{P V}$ (exp) and $A_{L L}^{P V}$. However, if we make the crude assumption that $A_{L L}=0$ (i.e. $P_{a} P_{b} A_{L L}=$ 0 and $\frac{1}{2} N^{00}=N^{--}+N^{++}=N^{-+}+N^{+-}$), and if we assume $P=P_{a}=P_{b}$, then we obtain the simple relation :

$$
A_{L L}^{P V}(\exp )=P A_{L L}^{P V} \longrightarrow \Delta A_{L L}^{P V}=\frac{1}{P} \sqrt{\frac{1-P^{2} A_{L L}^{P V}}{N^{--}+N^{++}}}
$$

We have suppressed the label "meas", because when there is a "simple relation" between the two asymmetries, the distinction between phenomenological simulations and experimental measurements, is no more required since they obey the same equation (here eq. (B.19)), even if physically the distinction between the two conditions still exists.

Finally, note that eq. $(B .19)$ is obtained under a crude assumption, but it exhibits the interesting property that the statistical errors of the PV spin asymmetries are $\sim 1 / P$. This has been already noted in [38], and in [48], where the formula eq.(B.17) has been also derived.

\section{B.3 Connections with some usual spin asymmetries}

The procedure described above is valid for any spin asymmetries. However, if for a certain asymmetry ( say $A$ ), there is a "simple relation" between the theoretical definition (i.e. A, $P$ independent) and the experimental definition (i.e. $A_{\text {exp }}, P$ dependent), it appears that the statistical error of the theoretical asymmetry has a simple expression. We examplify this fact for the two usual asymmetries $A_{L L}$ and $A_{\|}$. 
- $A_{L L}$

The $P$ dependent experimental asymmetry is defined by :

$$
A_{L L}^{e x p}=\frac{N_{e x p}^{++}-N_{e x p}^{+-}-N_{e x p}^{-+}+N_{e x p}^{--}}{N_{e x p}^{++}+N_{e x p}^{+-}+N_{e x p}^{-+}+N_{e x p}^{--}},
$$

associated to the statistical error :

$$
\Delta A_{L L}^{e x p}=\sqrt{\frac{1-A_{L L}^{e x p} 2}{N_{e x p}^{++}+N_{e x p}^{+-}+N_{e x p}^{-+}+N_{e x p}^{--}}} .
$$

Now, using the formula of correspondence (eq.(B.7)), we obtain the well-known simple formula : $A_{L L}^{e x p}=P_{a} P_{b} A_{L L}$.

Then, we deduce the statistical error for the theoretical $A_{L L}$ :

$$
\Delta A_{L L}=\frac{1}{P_{a} P_{b}} \cdot \Delta A_{L L}^{e x p}=\frac{1}{P_{a} P_{b}} \sqrt{\frac{1-A_{L L}^{e x p 2}}{N_{e x p}^{00}}}=\frac{1}{P_{a} P_{b}} \sqrt{\frac{1-P_{a}^{2} P_{b}^{2} A_{L L}^{2}}{N^{00}}}
$$

It is clear on this example, that it is no more necessary to distinguish the fact that we are performing a phenomenological analysis or analysing some experimental measurements. The equations are the same for the two cases (for the latter case and using our notations, we just have to add the subscript meas to all the preceding equations).

- $A_{\|}$

In general, in polarized deep inelastic scattering, rather than analysing the spin asymmetry $A_{L L}$ it is usual to study its reduced expression $A_{\|}\left(\equiv A_{2}^{2}\right.$ or $\left.A_{2}^{4}\right)$.

The $P$ dependent experimental asymmetry is defined by :

$$
A_{\|(\exp )}=\frac{N_{e x p}^{++}-N_{e x p}^{+-}}{N_{e x p}^{++}+N_{e x p}^{++}},
$$

associated to the statistical error :

$$
\Delta A_{\|(\exp )}=\sqrt{\frac{1-A_{\|}^{2}(e x p)}{N_{e x p}^{++}+N_{e x p}^{+-}}} .
$$

In terms of the "theoretical" number of events, we obtain :

$$
A_{\|(\exp )}=\frac{\frac{1}{2}\left(1+P_{a}\right) P_{b}\left[N^{++}-N^{+-}\right]-\frac{1}{2}\left(1-P_{a}\right) P_{b}\left[N^{--}-N^{-+}\right]}{\frac{1}{2}\left(1+P_{a}\right)\left[N^{++}+N^{+-}\right]+\frac{1}{2}\left(1-P_{a}\right)\left[N^{--}+N^{-+}\right]}
$$


We see, now, that there is no more a simple relation between $A_{\|}(\exp )$ and $A_{\|}$. However, if we assume parity conservation (i.e. $N^{++}=N^{--}$and $N^{+-}=N^{-+}$), we recover the well-known formula : $A_{\|}(\exp )=P_{a} P_{b} A_{\|}$, giving for the "theoretical" statistical error :

$$
\Delta A_{\|} \simeq \frac{1}{P_{a} P_{b}} \cdot \Delta A_{\|}(\exp )=\frac{1}{P_{a} P_{b}} \sqrt{\frac{1-P_{a}^{2} P_{b}^{2} A_{\|}^{2}}{N^{++}+N^{+-}}}
$$

If we do not assume parity conservation, eq. $(\overline{B .26})$ is no longer valid, and we have to use the procedure presented for $A_{L L}^{P V}$.

Finally, we have used a similar procedure in the one-spin case.

\section{References}

[1] E. Eichten, K. Lane and M. Peskin, Phys. Rev. Lett. 50, 811 (1983).

[2] E. Eichten et al., Rev. Mod. Phys. 56 (1984) 579.

[3] P. Haberl, F. Schrempp and H.-U. Martyn, p.1133 of [11.

[4] M.A. Doncheski, Z. Phys. C52 (1991) 527.

[5] V. Barger et al., Phys. Rev. D57 (1998) 391.

[6] P. Chiappetta and J.-M. Virey, Phys. Lett. B389 (1996) 89 .

[7] R. Rückl, Phys. Lett. B129 (1983) 363 and Nucl. Phys. B234 (1984) 91.

[8] R.J. Cashmore et al., Phys. Rep. 122 (1985) 275.

[9] H.-U. Martyn, p.801 of 10.

[10] 1987 HERA Workshop, Ed. R.D. Peccei.

[11] 1991 HERA Workshop, Eds. W. Buchmüller and G. Ingelman.

[12] HERMES Collaboration, K. Coulter et al., Proposal DESY-PRC 90/01 (1990); Technical Design Report DESY-PRC 93/06 (1993).

[13] J. Feltesse and A. Schäfer, p.757 of [14.

[14] 1996 HERA Workshop, "Future Physics at HERA", Eds. G. Ingelman, A. De Roeck and R. Klanner.

[15] "1997 Workshop on Physics with Polarized Protons at HERA", Eds. A. De Roeck and T. Gehrmann. 
[16] J.-M. Virey, p.152 of [15] (hep-ph/9710423); p.331 of the proceedings of the Workshop on physics beyond the Standard Model : "Beyond the Desert: Accelerator and Non-Accelerator Approaches", Eds. H.V. Klapdor-Kleingrothaus and H. Päs hepph/9707470).

[17] N. Di Bartolomeo and M. Fabbrichesi, Phys. Lett. B406 (1997) 237.

[18] A.E. Nelson, Phys. Rev. Lett. 78 (1997) 4159.

[19] A. Deandrea, Phys. Lett. B409 (1997) 277.

[20] G. 't Hooft, in Recent developments in Gauge Theories, Proceedings of the 1979 NATO Advanced Study Institute, Cargese, Eds. G. 't Hooft et al., (Plenum, New York), p.135.

[21] T. G. Rizzo et al., in Proceedings of the 1996 DPF/DPB Summer Study on New Directions for High Energy Physics (Snowmass 96), Snowmass CO, SLAC-Pub-7365, hep-ph/9612440; M. Cvetič and S. Godfrey, in Electroweak Symmetry Breaking and Beyond the Standard Model, eds. T. Barklow et al., World Scientific 1995.

[22] V. Barger et al., Phys. Rev. D57 (1998) 3833.

[23] C. Adloff et al. (H1), Z. Phys. C74 (1997) 191; J. Breitweg et al. (ZEUS), Z. Phys. C74 (1997) 207.

[24] G. Altarelli, CERN-TH-97-195, hep-ph/9708437, Nucl. Phys. Proc. Suppl. 62 (1998) 3 ; and references therein.

[25] V. Barger et al., Phys. Lett. B404 (1997) 147.

[26] B. Straub, Talk presented at LP'97, Hamburg, July 1997.

[27] A. Leike, preprint LMU-09-97, hep-ph/9708337.

[28] H1 Collaboration, S. Aid et al, Phys. Lett. B353 (1995) 578 ; ZEUS Collaboration, Proceeding of the XXXVIII International Conference on High Energy Physics, Warsaw, Poland, (1996).

[29] H.-U. Martyn, private communication.

[30] M. Glück et al., Z. Phys. C67 (1995) 433.

[31] A.D. Martin et al., Phys. Lett. B354 (1995) 155.

[32] C. Bourrely and J. Soffer, Nucl. Phys. B445 (1995) 341.

[33] M. Glück et al., Phys. Rev. D53 (1996) 4775.

[34] T. Gehrmann and W.J. Stirling, Phys. Rev. D53 (1996) 6100; 
[35] RHIC Spin Collaboration, Letter of intent, April 1991; G. Bunce et al., Polarized protons at RHIC, Particle World 3 (1992) 1; Proceedings of the RSC annual meeting, Marseille, September 1996, preprint CPT-96/P.3400; Proceedings of RIKEN-BNL Research Center Workshop, "RHIC Spin Physics", April 1998, preprint BNL-65615.

[36] J. Soffer and J.-M. Virey, Nucl. Phys. B509 (1998) 297; and references therein.

[37] P. Taxil and J.-M. Virey, Phys. Lett. B364, (1995) 181.

[38] P. Taxil and J.-M. Virey, Phys. Rev. D55, (1997) 4480.

[39] C. Bourrely et al., Phys. Rep. 177 (1989) 319.

[40] H.-U. Martyn, "Perspectives on new phenomena" in [14].

[41] R.D. Ball et al., p.777 of [14]; A. De Roeck et al., preprint DESY-97-249, hep$\mathrm{ph} / 9801300$.

[42] Particle Data Group, Phys. Rev. D54 (1996) 1.

[43] C. Y. Prescott et al., Phys. Lett. B77 (1978) 347 ; Phys. Lett. B84 (1979) 524.

[44] H.-U. Martyn et al., p.987 of [11].

[45] P. Haberl et al., p.980 of [11].

[46] P. Taxil, E. Tuğcu and J.-M. Virey, in preparation.

[47] C. Bourrely, J.-Ph. Guillet and J. Soffer, Nucl. Phys. B361 (1991) 72.

[48] B. Kamal, Phys. Rev. D57 (1998) 6663. 